\title{
Article \\ Selection of Output Voltage Compensators Gains in Two Cascaded Boost Converters with Input Filters by Means of the $\mathfrak{D}$-Decomposition Technique
}

\author{
Karol Najdek*(D), Radosław Nalepa (D) and Robert Lis (D) \\ Department of Electrical Power Engineering, Faculty of Electrical Engineering, \\ Wrocław University of Science and Technology, 50370 Wrocław, Poland; radoslaw.nalepa@pwr.edu.pl (R.N.); \\ robert.lis@pwr.edu.pl (R.L.) \\ * Correspondence: karol.najdek@pwr.edu.pl; Tel.: +48-71-3202-135
}

Citation: Najdek, K.; Nalepa, R.; Lis, R. Selection of Output Voltage Compensators Gains in Two Cascaded Boost Converters with Input Filters by Means of the $\mathfrak{D}$-Decomposition Technique. Energies 2021, 14, 5883. https:// doi.org/10.3390/en14185883

Academic Editor: Adolfo Dannier

Received: 5 August 2021

Accepted: 13 September 2021

Published: 17 September 2021

Publisher's Note: MDPI stays neutral with regard to jurisdictional claims in published maps and institutional affiliations.

Copyright: (C) 2021 by the authors. Licensee MDPI, Basel, Switzerland. This article is an open access article distributed under the terms and conditions of the Creative Commons Attribution (CC BY) license (https:// creativecommons.org/licenses/by/ $4.0 /)$.

\begin{abstract}
In this paper, the $\mathfrak{D}$-decomposition technique is investigated as an intuitive method for finding the non-linear trajectories of PI-compensator gains. The trajectories reflect the desired dynamic properties at a system level specified by the gain and the phase margin (GMPM) in the frequency domain. They are presented as parametric curves in the proportional and the integral gains coordinates in form of $K_{\mathrm{I}}=f\left(K_{\mathrm{P}}\right)$ functions. The curves are inscribed into global stability boundaries (GSB). The corresponding Nyquist plots are included for comparison. The analysis is based on a system consisting of two serial-connected boost converters. Each converter has its input filter. The major parasitic components of the system are taken into account during the mathematical and simulation modelling. The control circuit time delays and non-linear semiconductors characteristics are also included. A complete set of practically useful system-level transfer functions in form of mathematical formulas is included. Selected aspects, such as the control-to-output voltage and the control-to-input current of one sub-system of the simulation model, have been verified experimentally. The presented results clearly indicate the need for interactions between the sub-systems of a system to be taken into account during controller gains selection.
\end{abstract}

Keywords: $\mathfrak{D}$-decomposition technique; boost converter; cascaded converters; PI voltage compensator; stability boundary

\section{Introduction}

Power electronics systems (PES), understood as power electronics circuits (PEC) working together, are gaining popularity as modular solutions for electric power conversion [1,2]. They are commonly used in the AC, DC or combined AC-DC installations. Nevertheless, in terms of the PES control design, the level of complexity is higher when compared to a single PEC. It is driven by necessity of taking into account interactions between connected PECs. The connections may be of serial and/or parallel combinations of inputs and/or outputs [3]. Such a variety of options makes it even more challenging. There is no simple control structure block diagram such as, for example, the classical negative feedback singleloop scheme. Therefore, the PES control design requires new, intuitive solutions. Such an approach can be developed based on a comprehensive understanding of system-level interactions and controlled devices. In principle, the approach could rely on a selection of parameters and controller gains, ensuring the fulfilment of selected system-level control criteria [4,5]. Of course, the roots of the solutions are deeply on the basis of the PID or PI controllers tuning for PEC [6,7]. There are also existing system-level advanced solutions deeply based on fuzzy logic [8,9] or artificial neural networks with a quasi-network back propagation algorithm [10], but these approaches belong to numerical optimization methods. For numerical methods, particular solutions are identified without direct and unambiguous indication of the dynamic properties. 
Desired, physically possible, dynamic properties of PES can be obtained using an advanced approach, but selection of the controller parameters is more complex than in classical techniques dedicated to PEC. For this reason, methods based on modified classical approaches are often used. A good example is a controller based on adaptation of its gains, so-called gain scheduling [11,12]. However, use of such a scheme may be challenging during the adaptation curve selection in a controller with a wide operating range.

An attractive solution could be the use of graphical methods that are more intuitive during complex analyses. They lead to visualization of the compensator gains trajectories in function of selected parameters. One such method is the $\mathfrak{D}$-decomposition technique, presented in 1948 by Russian mathematician Yuri Issakovich Neimark [13]. The $\mathfrak{D}$-decomposition technique, called known as D-partition, establishes a direct correlation between the characteristic equation and the space of permissible parameters for which the global stability is met. The technique in its original form indicates only the GSB in form of $K_{\mathrm{I}}=f\left(K_{\mathrm{P}}\right)$ plot for a PI compensator [14-16]. In case of the PID regulator, the differential gain appears as a parameter.

The $\mathfrak{D}$-decomposition technique can be relatively easily modified to take into account selected constraints such as the two fundamental constraints in the frequency domain, the gain margin (GM) and the phase margin (PM) [14,17]. It also can be adapted to the Maximum Sensitivity (Ms) [18] or even time domain constraints such as the overshoot and the rise time [18,19]. Furthermore, the GM, PM and Ms can be combined with the time domain constraints such as the overshoot and the rise time [18].

An alternative solution to the $\mathfrak{D}$-decomposition technique could be an approach based on the Lyapunov theory [20]. However, it is less intuitive when compared to the $\mathfrak{D}$-decomposition applied even to a complex systems such as a two-mass drive [21]. Despite the relative complexity, the Lyapunov function is also used at the system-level. In [22], it is applied to ensure the stable operation of an observer of a sub-system consisting of a DC converter with an input filter.

The impedance-based methods used at the level of individual PEC are also used at the system level [23]. Nevertheless, in their basic form, they do not take into account the system-level interactions. In case of connecting a few PEC into a group, the interactions between them must be taken into account during selection of the controllers gains. Properly chosen gains for standing alone units do not guarantee the stable operation of a system containing a group of them working together.

In case of the system-level considerations, Nyquist characteristics are also used. They are drawn for assumed gains based on the system transfer functions [24]. Here, the transfer functions of converters are often represented by a two-port network [25]. The Nyquist characteristics are used for verifying selected controller gains instead of searching for them. In such cases dedicated system criteria are used with the plots. The criteria such as the Middlebrook, the Gain Margin and Phase Margin (GMPM), the Opposing Argument (OA), Energy Source Analysis Consortium (ESAC) and the Maximum Peak Criterion (MPC) [24] allow for formation of the forbidden regions within the Nyquist plots. The Nyquist plots are also used in the analysis of changes in selected parameters and interactions between the devices [23]. Such an approach requires the analysis of many Nyquist plots. Then, on this basis, one can determine the minimum value of the selected system constraints. Consequently, it becomes quite a complex process.

The motivation to do this work was to use the $\mathfrak{D}$-decomposition technique and the GMPM system criteria to indicate output voltage controller gains of a known and relatively simplepower electronics circuit being a part of the cascaded system. Here, the boost converter seems to be a good candidate. This popular topology is often employed in industrial applications as a single PEC [26] and as a part of a PES [22,24,27]. It can also be a part of a more advanced reconfigurable circuit like one described in [28] Despite of its simple topology, it represents a challenge in terms of the control because of its controlto-output transfer function right-half plane zero [29]. Of course, such a topology in many applications also requires a current control loop or at least some sort of current limiting [30]. 
This paper investigates the application of GMPM stability criteria combined with the $\mathfrak{D}$-decomposition technique for series-connected two DC-DC boost converters. Each converter contains an LC input filter. Optimized design of the filters was not an objective of this research. For simplicity, the connection line between the converters was not taken into account. The proposed approach is based on small-signal modelling with major parasitic components taken into account. All the transfer functions have been confirmed in simulation by means of identification. The identified control-to-output voltage and controlto-input current transfer functions of the first converter have been verified experimentally. The provided mathematical formulas constitute a complete set of equations ready to be used with any other cascaded system consisting of two boost converters with input filters as subsystems. The controller gains selection path is shown step-by-step. It starts with individual converter gains selection before connecting them into one system. The obtained results are compared against the output converter performance with gains optimized by taking into account interactions at the system-level. The results undisputedly show need for interactions to be taken into account during the control tuning of a power electronics circuit connected to an existing power electronics circuit.

The key features of this article are:

- $\quad$ intelligible indication of a path towards systematic design of PES control solutions based on an example of a two serial connected boost converters in the voltage mode control,

- $\quad$ use of the $\mathfrak{D}$-decomposition technique not only for the system-level stability boundaries calculation but also for indication of gains region fulfilling particular GMPM requirements.

The paper is organized in six sections. As an introduction, the reasons for this work are presented. In the next section, configuration of the PES has been presented, followed by details on the subsystem-level transfer functions. After that, the system-level dependencies are formulated. Once all the transfer functions are ready, the $\mathfrak{D}$-decomposition technique is used for indication of suitable gains trajectories taking into account the GMPM criteria. The results are compared and discussed in this section. The conclusions are given in the last section.

\section{Configuration of the PES Considered}

For purpose of explanation a PES configutarion as shown in Figure 1 was used. The 1 st sub-circuit or sub-system, sub1, contains a passive $L C$ input filter with parameters listed in Table 1. For simplicity, this is exactly the same as sub3. The filters are taken into account together with their basic parasitic components, such as the $r_{\mathrm{LF}}$ and the $r_{\mathrm{CF}}$. They stand for the equivalent series resistances of the inductance and the capacitance, respectively. The remaining sub-systems, sub2 and sub4, contain Boost converters as output voltage controlled PEC. Their parameters can bee seen in Table 2. The sub2 and sub4 consist of the same components. Their output powers are $P_{\text {sub2,out }}^{\text {rtd }}=P_{\text {sub4,out }}^{\text {rtd }}=108 \mathrm{~W}$. The only differences are the input voltages, $V_{\text {sub2,in }}^{\text {rtd }}=30 \mathrm{~V}$ and $V_{\text {sub4,in }}^{\text {rtd }}=45 \mathrm{~V}$, and the output voltages, $V_{\text {sub2,out }}^{\text {rtd }}=45 \mathrm{~V}$ and $V_{\text {sub4,out }}^{\text {rtd }}=60 \mathrm{~V}$, respectively. Parasitic components such as the esr and the esl were taken into account too. The converter was designed to operate at constant switching frequency, $f_{\mathrm{s}}=20 \mathrm{kHz}$, in the Continuous Conduction Mode (CCM). The design relied on MATALB/Simulink environment. For simplicity, only the output voltages were controlled and without the output currents control. The closed loop voltage control relies on the standard structure shown in Figure 2. Here, the low-pass filter in the feedback loop is omitted as its bandwidth was set away, $80 \mathrm{kHz}$, from the $20 \mathrm{kHz}$ sampling frequency. In case of presence of the current control loop (as an inner loop), it would have to be tuned before the outer voltage controller. Then, after that, the voltage loop equations would be formulated taking into account the inner structure. This approach will be presented in a future article. 


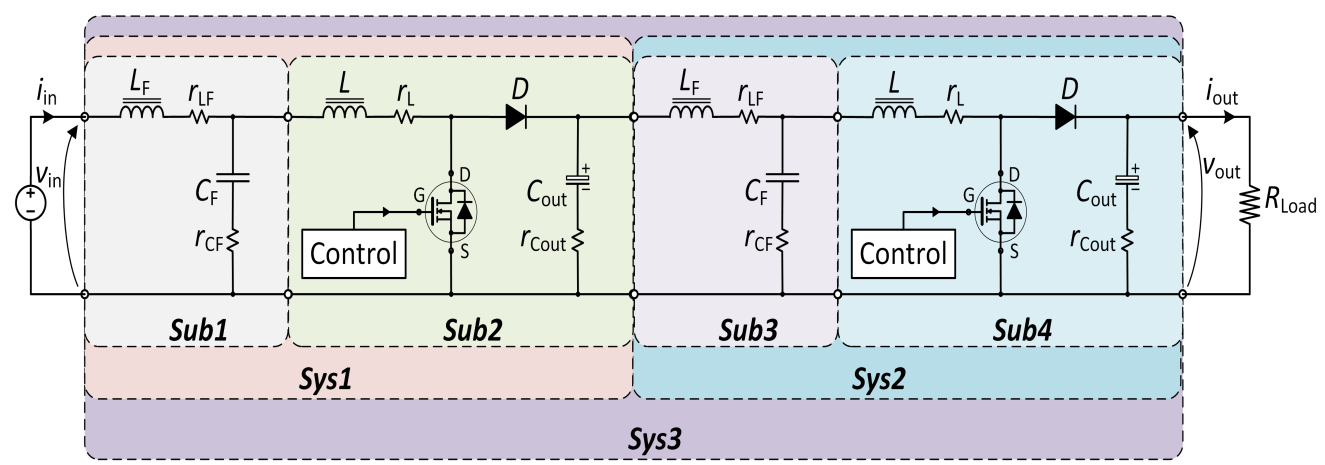

Figure 1. Equivalent circuit diagram of two cascaded boost converters considered as sub-circuits $s u b 2$ and $s u b 4$, with their $L C$ input filters as sub-circuits sub1 and sub3, respectively. The sub1 and $s u b 2$ together constitute a system sys1. The $s u b 3$ and sub4 constitute a system sys 2 . Serial connection of the sys 1 and the sys 2 stands for a sys 3 understood here as the top level system. The sub2 and sub4 are sub-circuits under control. The parasitics, such as inductors $L_{\mathrm{F}}$ and $L$ equivalent series resistances $r_{\mathrm{LF}}$ and $r_{\mathrm{L}}$, respectively, and capacitors $C_{\mathrm{F}}$ and $C_{\text {out }}$ equivalent series resistances $r_{\mathrm{CF}}$ and $r_{\mathrm{C}_{\text {out }}}$, have been taken into account.

For simplicity, the system sys3 shown in Figure 1 does not take into account connecting line impedance between the sys 1 and the sys 2 . It assumes a direct connection between the systems.

Table 1. Parameters of the filters sub-circuits, sub1 and sub3, of the system sys3 shown in Figure 1.

\begin{tabular}{ccccc}
\hline Parameter Name & Symbol & Value in sub1 & Value in sub3 & Unit \\
\hline Filter inductance & $L_{\mathrm{F}}$ & 1 & 10 & $\mu \mathrm{H}$ \\
The inductance esr & $r_{\mathrm{LF}}$ & 50 & 50 & $\mathrm{~m} \Omega$ \\
Filter capacitance & $C_{\mathrm{F}}$ & 1 & 0.1 & $\mathrm{mF}$ \\
The capacitor esr & $r_{\mathrm{CF}}$ & 10 & 10 & $\mathrm{~m} \Omega$ \\
\hline
\end{tabular}

Table 2. Parameters of the Boost converters, sub2 and sub4, of the system sys 3 shown in Figure 1.

\begin{tabular}{|c|c|c|c|}
\hline Parameter Name & Symbol & Value & Unit \\
\hline Rated output power & $P_{\text {sub2,out }}^{\text {rtd }} P_{\text {sub4,out }}^{\text {rtd }}$ & 108 & W \\
\hline Rated input voltage of $s u b 2$ & $V_{\text {sub2 in }}^{\text {rtd }}$ & 30 & $\mathrm{~V}$ \\
\hline Rated output voltage of $s u b 2$ & $V_{\text {sub2,out }}^{\text {rtd }}$ & 45 & $\mathrm{~V}$ \\
\hline Rated input voltage of sub4 & $V_{\text {sub4,in }}^{\text {rtd, }}$ & 45 & $\mathrm{~V}$ \\
\hline Rated output voltage of $s u b 4$ & $V_{\text {sub4,out }}^{\text {rtd }}$ & 60 & $\mathrm{~V}$ \\
\hline Switching frequency of $s u b 2$ and $s u b 4$ & $f_{\mathrm{S}}$ & 20 & $\mathrm{kHz}$ \\
\hline Steady state duty cycle of $s u b 2$ at $P_{\text {sub2,out }}^{\text {rtd }}$ & $D_{\text {sub2 }}$ & 0.35 & - \\
\hline Steady state duty cycle of $s u b 4$ at $P_{\text {sub4,out }}^{\text {rtd }}$ & $D_{\text {sub4 }}$ & 0.262 & - \\
\hline Boost inductance of $s u b 2$ and $s u b 4$ & $\mathrm{~L}$ & 283 & $\mu \mathrm{H}$ \\
\hline The Boost inductance esr & $r_{\mathrm{L}}$ & 150 & $\mathrm{~m} \Omega$ \\
\hline Boost output capacitance & $C_{\text {out }}$ & 470 & $\mu \mathrm{F}$ \\
\hline The Boost output capacitors esr & $r_{\mathrm{C}_{\text {out }}}$ & 350 & $\mathrm{~m} \Omega$ \\
\hline
\end{tabular}

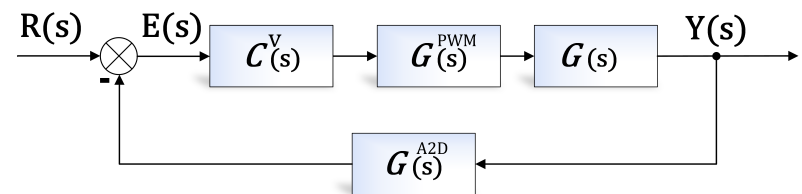

Figure 2. Simplified block diagram of the closed loop control structure with the PWM and the A2D delays represented by the $G^{\mathrm{PWM}}(s)$ and $G^{\mathrm{A} 2 \mathrm{D}}(s)$ transmitances, respectively. The plant under control transfer function is represented by the $G(s)$ while the $C^{\mathrm{v}}(s)$ stands for a controller. 


\section{Transfer Functions of the PES}

In order to conduct system-level analyses of the systems shown in Figure 1, transfer functions of each of the subsystems have been mathematically calculated. They are marked with superscript annotation . mat,c —as, a different notation from simulation . ${ }^{\text {sim,., }}$

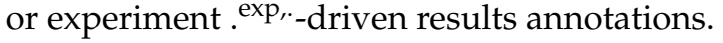

\subsection{The sub2 and sub4 Boost Converters}

The Boost converters from the sub2 and sub4 as controlled PEC can be described by transfer functions derived based on the small signal analysis [2,31], and represented by Equations (1)-(6). In these equations the output resistance $R_{\text {out }}$ represents the converter load. The equations are the same for sub2 and sub4 and, therefore, for simplicity, \# is used instead of 2 or 4 indexes.

$$
G_{\text {sub\# }}^{\text {mat, },, \text { vin }_{\text {in }} 2 \mathrm{v}_{\text {out }}}(s)=\left.\frac{\widetilde{v}_{\text {sub\#, out }}(s)}{\widetilde{v}_{\text {sub\#, in }}(s)}\right|_{\widetilde{d}(s), \widetilde{i}_{\text {subf }, \text { out }}(s)=0}=M_{\mathrm{vx}} \frac{s+\omega_{\text {zn }}}{s^{2}+2 \xi \omega_{\mathrm{n}} s+\omega_{\mathrm{n}}^{2}}
$$

where: $M_{\mathrm{vx}}=\frac{{ }^{r} \mathrm{C}_{\text {out }} R_{\text {out }}(1-D)}{L\left(r_{\mathrm{C}_{\mathrm{out}}}+R_{\mathrm{out}}\right)}$.

$$
G_{\text {sub\# }}^{\operatorname{mat}, c, \text { vin }_{\text {in }} 2 \mathrm{i}_{\text {in }}}(s)=\left.\frac{\widetilde{i}_{\text {sub\#,in }}(s)}{\widetilde{v}_{\text {sub\#,in }}(s)}\right|_{d(s), \tilde{i}_{\text {sub\#, out }}(s)=0}=M_{\mathrm{vix}} \frac{s+\omega_{\text {zi } 2}}{s^{2}+2 \tilde{\xi} \omega_{\mathrm{n}} s+\omega_{\mathrm{n}}^{2}}
$$

where: $M_{\mathrm{vix}}=\frac{1}{L}$.

$$
\begin{aligned}
& \omega_{\text {zi2 }}=\frac{1}{C_{\text {out }}\left(R_{\text {out }}+r_{\mathrm{C}_{\text {out }}}\right)} \\
& G_{\text {sub\# }}^{\text {mat, }, \dot{\mathrm{i}}_{\text {out }} 2 \mathrm{v}_{\text {out }}}(s)=\left.\frac{\widetilde{v}_{\text {sub\#,out }}(s)}{-\widetilde{i}_{\text {sub\#, out }}(s)}\right|_{\widetilde{v}_{\text {sub\#,in }}(s), \widetilde{d}(s)=0}=Z_{\mathrm{ox}} \frac{\left(s+\omega_{\mathrm{rl}}\right)\left(s+\omega_{\mathrm{zn}}\right)}{s^{2}+2 \tilde{\xi} \omega_{\mathrm{n}} s+\omega_{\mathrm{n}}^{2}}
\end{aligned}
$$

where: $Z_{\mathrm{ox}}=\frac{r_{\mathrm{C}_{\text {out }}} R_{\text {out }}}{R_{\text {out }}+{ }^{r_{\mathrm{Cut}}}}$

$$
\begin{aligned}
& \omega_{\text {rl }}=\frac{r_{\mathrm{L}}}{L} \\
& \omega_{\text {zn }}=\frac{1}{C_{\text {out }} \mathrm{C}_{\text {out }}} \\
& \omega_{\mathrm{n}}=\sqrt{\frac{(1-D)^{2} R_{\text {out }}+r_{\mathrm{L}}}{L C_{\text {out }}\left(R_{\text {out }}+r_{C_{\text {out }}}\right)}} \\
& \xi=\frac{C_{\text {out }}\left[r_{\mathrm{L}}\left(R_{\text {out }}+r_{C_{\text {out }}}\right)+R_{\text {out }} C_{\text {out }}(1-D)^{2}\right]+L}{2 \sqrt{L C_{\text {out }}\left(R_{\text {out }}+r_{C_{\text {out }}}\right)\left[r_{\mathrm{L}}+(1-D)^{2} R_{\text {out }}\right]}} . \\
& \quad G_{\text {sub\# }}^{\text {mat }, i_{\text {out }} 2 i_{\text {in }}}(s)=\left.\frac{\widetilde{i}_{\text {sub\#, in }}(s)}{\widetilde{i}_{\text {sub\# }, \text { out }}(s)}\right|_{d(s), \widetilde{i}_{\text {sub\# }, \text { out }}(s)=0}=A_{\text {ix }} \frac{s+\omega_{\text {zn }}}{s^{2}+2 \xi \omega_{\mathrm{n}} s+\omega_{\mathrm{n}}^{2}}
\end{aligned}
$$

where: $A_{\mathrm{ix}}=\frac{R_{\text {out }}{ }^{r} \mathrm{C}_{\text {out }}(1-D)}{L\left(R_{\text {out }}+r_{\mathrm{C}_{\text {out }}}\right)}$.

$$
G_{\text {sub\# }}^{\text {mat, }, \mathrm{d} 2 \mathrm{v}_{\text {out }}}(s)=\left.\frac{\widetilde{v}_{\text {sub\#, out }}(s)}{\widetilde{d}(s)}\right|_{\widetilde{v}_{\text {sub\#, in }}(s), \widetilde{i}_{\text {subf }, \text { out }}(s)=0}=T_{\mathrm{px}} \frac{\left(s-\omega_{\mathrm{zp}}\right)\left(s+\omega_{\mathrm{zn}}\right)}{s^{2}+2 \tilde{\xi} \omega_{\mathrm{n}} s+\omega_{\mathrm{n}}^{2}}
$$

where: $T_{\mathrm{px}}=\frac{-{ }^{-} \mathrm{C}_{\text {out }} V_{\text {out }}}{\left(R_{\text {out }}+{ }^{+} \mathrm{C}_{\text {out }}\right)(1-D)}$. 


$$
\begin{aligned}
\omega_{\text {zp }}= & \frac{R_{\text {out }}(1-D)^{2}-r_{\mathrm{L}}}{L} \\
& G_{\text {sub\# }}^{\text {mat,c,d } 22_{\text {in }}}(s)=\left.\frac{\widetilde{i}_{\text {sub\#,in }}(s)}{\widetilde{d}(s)}\right|_{\widetilde{v}_{\text {sub\#, }, \text { in }, \widetilde{i}_{\text {sub\# }, \text { out }}(s)=0}}=T_{\text {pix }} \frac{s+\omega_{\text {zi }}}{s^{2}+2 \widetilde{\zeta} \omega_{\mathrm{n}} s+\omega_{\mathrm{n}}^{2}}
\end{aligned}
$$

where: $T_{\text {pix }}=\frac{V_{\text {out }}\left(R_{\text {out }}+2 r_{\mathrm{C}_{\text {out }}}\right)}{L\left(R_{\text {out }}+r_{\mathrm{C}_{\text {out }}}\right)}, \omega_{\mathrm{zi}}=\frac{1}{\mathrm{C}_{\mathrm{out}}\left(R_{\text {out }}\right)}$.

The Equations (1)-(6) with numeric values from Table 2 and $R_{\text {out }}=33.3 \Omega$ take following form for the sub2:

$$
\begin{aligned}
& G_{\text {sub2 }}^{\text {mat, }, \text {, } V_{\text {in }} 2 v_{\text {out }}}(s)=\frac{341.8 s+4.848 \times 10^{6}}{s^{2}+718.7 s+3.194 \times 10^{6}} \\
& G_{\text {sub2 }}^{\text {mat, }, \text {, } \text { in }_{\text {in }} 2 i_{\text {in }}}(s)=\frac{3.534 s+3.978 \times 10^{5}}{s^{2}+718.7 s+3.194 \times 10^{6}} \\
& G_{\text {sub2 }}^{\text {mat, }, \mathrm{i}_{\text {out }} 2 \mathrm{v}_{\text {out }}}(s)=\frac{0.1488 s^{2}+2168 s+8.105 \times 10^{5}}{s^{2}+718.7 s+3.194 \times 10^{6}} \\
& G_{\text {sub2 }}^{\text {mat, }, \dot{i}_{\text {out }} 22_{\text {in }}}(s)=\frac{341.8 s+4.848 \times 10^{6}}{s^{2}+718.7 s+3.194 \times 10^{6}} \\
& G_{\text {sub2 }}^{\text {mat,c,d2 }} \text { out }(s)=\frac{-0.5495 s^{2}+7376 s+2.152 \times 10^{8}}{s^{2}+718.7 s+3.194 \times 10^{6}} \\
& G_{\text {sub2 }}^{\text {mat, }, \text { d } 2 i_{\text {in }}}(s)=\frac{1.603 \times 10^{5} s+3.58 \times 10^{7}}{s^{2}+718.7 s+3.194 \times 10^{6}}
\end{aligned}
$$

Similarly the sub4 equations can be calculated as following:

$$
\begin{aligned}
& G_{\text {sub4 }}^{\text {mat, }, v_{\text {in }} 2 v_{\text {out }}}(s)=\frac{389.4 s+5.524 \times 10^{6}}{s^{2}+736.1 s+4.101 \times 10^{6}} \\
& G_{\text {sub4 }}^{\text {mat, }, v_{\text {in }} 2 \mathrm{i}_{\text {in }}}(s)=\frac{3.534 s+2.246 \times 10^{5}}{s^{2}+736.1 s+4.101 \times 10^{6}} \\
& G_{\text {sub4 } 4}^{\text {mat, }, i_{\text {out }} 2 v_{\text {out }}}(s)=\frac{0.1493 s^{2}+2176 s+8.158 \times 10^{5}}{s^{2}+736.1 s+4.101 \times 10^{6}} \\
& G_{\text {sub4 } 4, \text { i }}^{\text {mat } 2 \mathrm{i}_{\text {in }}}(s)=\frac{389.4 s+5.524 \times 10^{6}}{s^{2}+736.1 s+4.101 \times 10^{6}} \\
& G_{\text {sub4 }}^{\text {mat,c,d2 }} v_{\text {out }}(s)=\frac{-0.3643 s^{2}+1.806 \times 10^{4} s+3.294 \times 10^{8}}{s^{2}+736.1 s+4.101 \times 10^{6}} \\
& G_{\text {sub4 }}^{\operatorname{mat}, c, d 2 i_{\text {in }}}(s)=\frac{2.13 \times 10^{5} s+2.695 \times 10^{7}}{s^{2}+736.1 s+4.101 \times 10^{6}}
\end{aligned}
$$

It should be noted that Equations (7)-(18) rely on the output power represented by the load resistance of the converter. In this case, the $P_{\text {sub2, out }}^{\text {rtd }}=P_{\text {sub4, out }}^{\text {rtd }}=108 \mathrm{~W}$ was used. The transfer functions represented by the Equations (7)-(12) result in the Bode plots shown in Figure 3. Two of the six transfer functions, the $G_{\text {sub2 }}^{\text {exp, } m, d 2 v_{\text {out }}}(s)$ and $G_{\text {sub2 }}^{\text {exp, } m, d 2 i_{\text {in }}}(s)$, were verified experimentally, see Figure $3 \mathrm{e}-\mathrm{f}$, respectively (the blue dots). The numerical transfer functions based on previously derived mathematical formulas are for nominal parameters only. In engineering practice the parameters tolerance is to be taken into account too. It leads to results in form of envelopes instead of single lines. Such an example envelope can be seen in Figure 3a. The grey areas are for $\pm 10 \%$ tolerance of the $L$ and $C_{\text {out }}$ and $\pm 50 \%$ of the $r_{\mathrm{L}}$ and $r_{\mathrm{C}_{\text {out }}}$. The outer envelope lines are then to be used during analysis. Nevertheless, 
here in this article, for simplicity, we concentrate only on the nominal parameters which are equivalent to values in simulation.

(a)

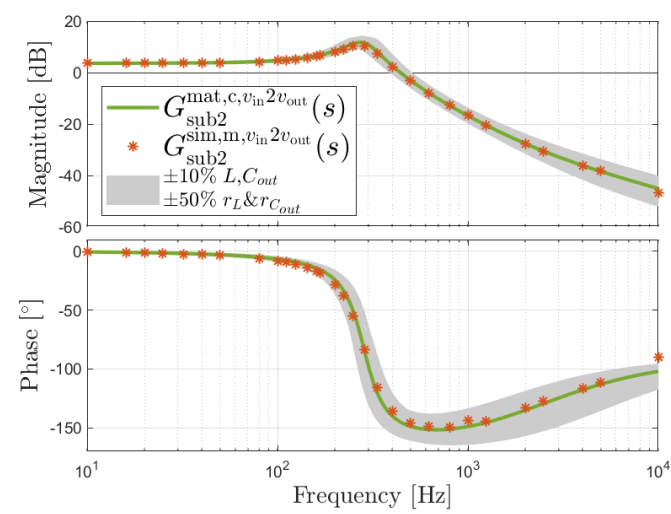

(c)

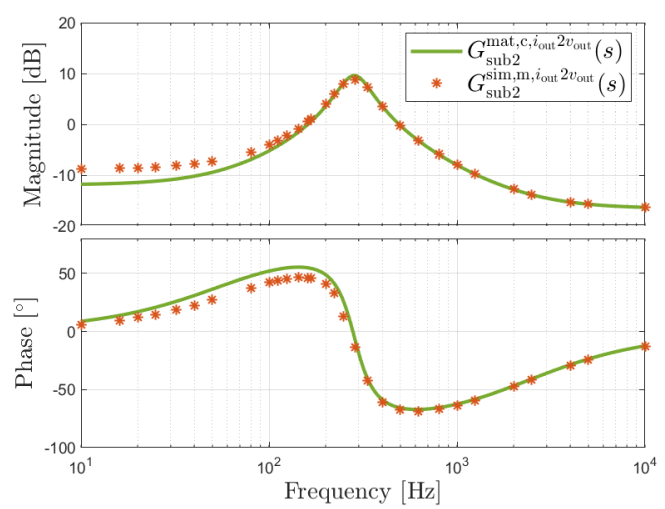

(e)

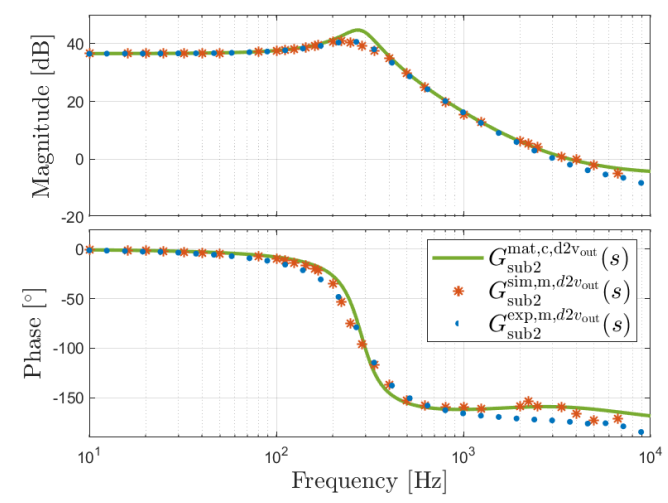

(b)

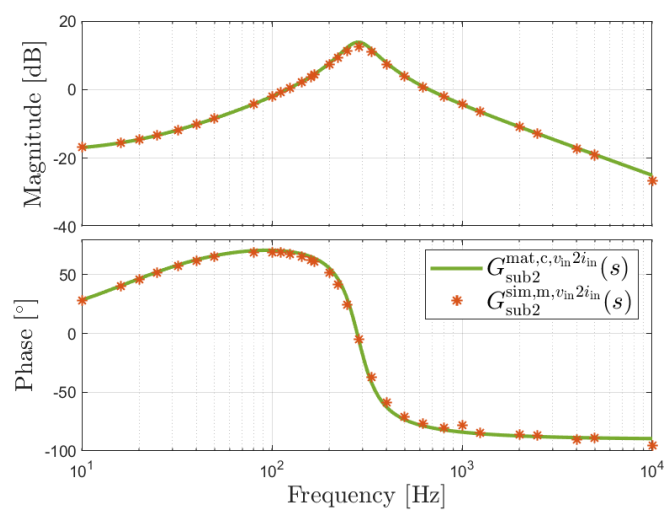

(d)

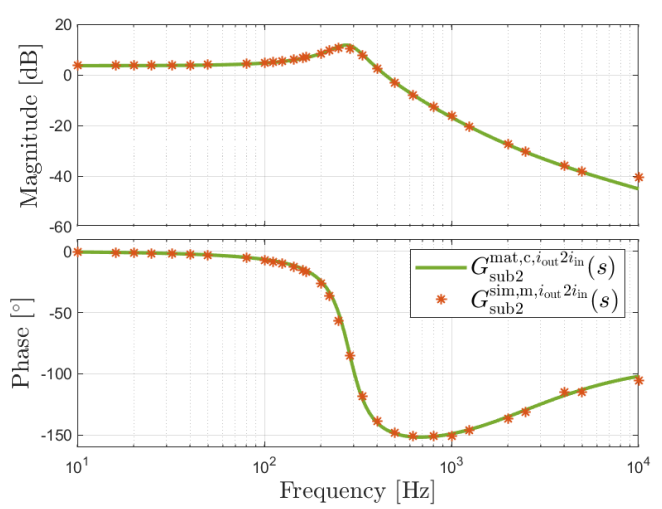

(f)

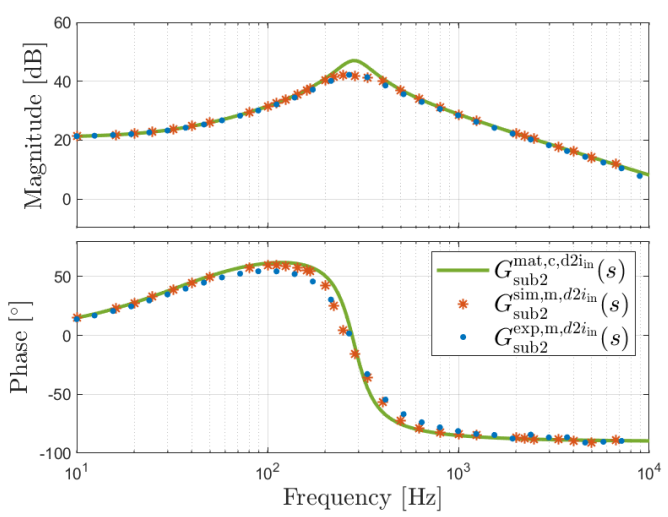

Figure 3. The Bode plots of the $s u b 2$ mathematically derived $G_{\text {sub2 } 2}^{\text {mat,c.2. }}(s)$ and simulation measurement confirmed $G_{\mathrm{sub} 2}^{\mathrm{sim}, \mathrm{m}, 2 .}(s)(\mathbf{a}-\mathbf{f})$ together with experimentally measured $G_{\mathrm{sub} 2}^{\mathrm{exp}, \mathrm{m}, 2 .}(s)(\mathbf{e}, \mathbf{f})$ : (a) voltage transmittance $G_{\text {sub2 }}^{\text {mat, }, v_{\text {in }} 2 v_{\text {out }}}(s)$ according to Equations (1) and (7); (b) input admittance $G_{\text {sub2 }}^{\text {mat,c, } v_{\text {in }} 2 \mathrm{i}_{\text {in }}}(s)$ according to Equations (2) and (8); (c) output impedance $G_{\text {sub2, }}^{\text {mat, } i_{\text {out }} 2 v_{\text {out }}}(s)$ according to Equations (3) and (9); (d) current transmittance $G_{\text {sub2 }}^{\text {mat, }, i_{\text {out }} 2 i_{\text {in }}}(s)$ according to Equations (4) and (10); (e) control transmitance $G_{\text {sub2 }}^{\text {mat,c,d2 }}$ out $(s)$ according to Equations (5) and (11); (f) control transmitance $G_{\text {sub2 }}^{\text {mat,c,d2i }}$ in $(s)$ according to Equations (6) and (12).

The boost converter from the $s u b 2$ has been physically built and tested in laboratory, see Figure 4. Its control was implemented with the TMS320F28379D development board. 
As the switching component CoolMOS power MOSFET IXKN 75N60C, switched at $20 \mathrm{kHz}$, has been used. The diode type was IXYS DSP25-12A. Such experimental validation has been done just to make sure that there is no mistakes in the mathematical modelling. Validation of the selected transfer functions does not require advanced setup for current or voltage injection. It relies on the duty cycle variation only (this around a certain operating point) and observation of the output voltage. Obtained experimental results confirm sufficient accuracy of the mathematical modelling.

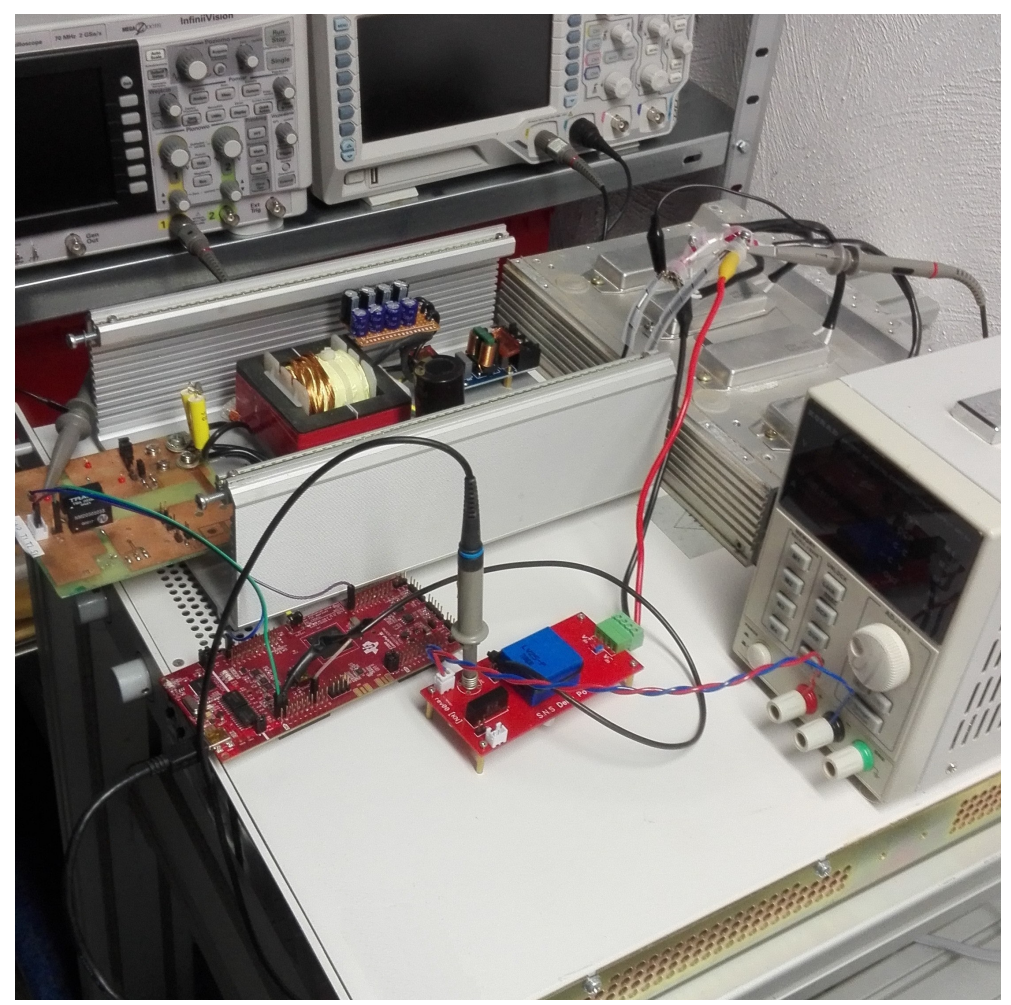

Figure 4. Experimental setup of a boost converter used for analysis conducted in this article. The circuit corresponds to a circuit diagram shown in Figure 2 with parameters as per Table 2.

Similarly the Bode plots of Equations (13)-(18) can be seen in Figure 5. In this case experimental validation was not conducted. Nevertheless, all the results shown in Figure 3 and 5 have been confirmed in simulation model taking into account the parasitic components shown in Figure 1 together with non-linearity of the voltage-current characteristic of the MOSFET. By the results comparison one can see that there is good agreement between the simulation and the mathematical results. Visible, although acceptable, differences in certain frequency ranges are driven by simplifications in the mathematical formulas. The simulation results from Figure $3 \mathrm{e}, \mathrm{f}$ are in a very satisfactory accordanceto the experimentally recorded points. 
(a)

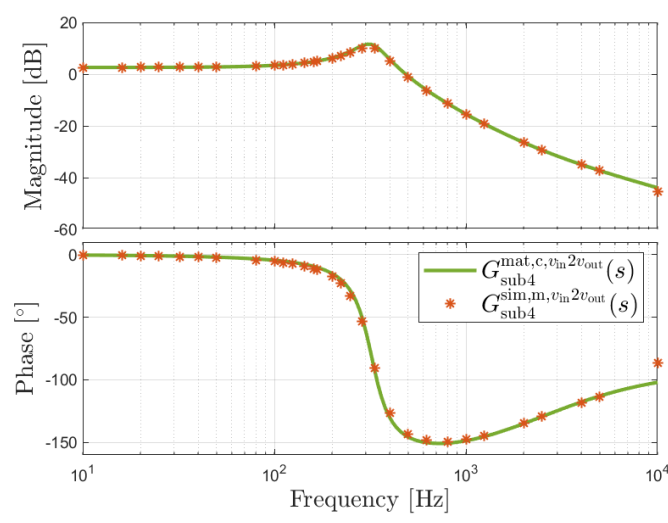

(c)

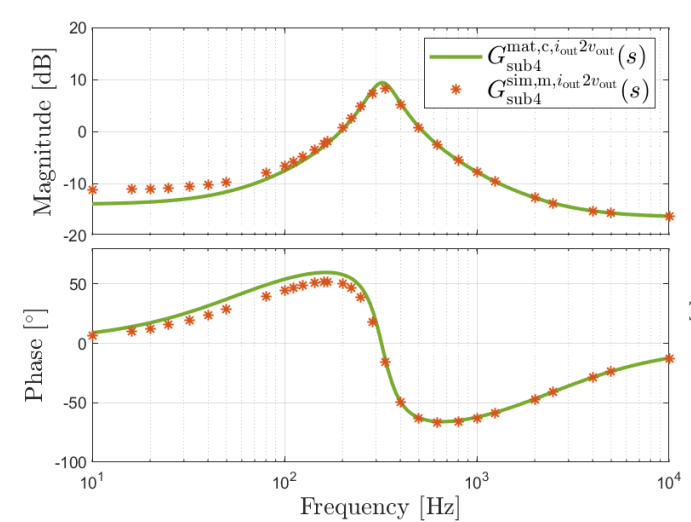

(e)

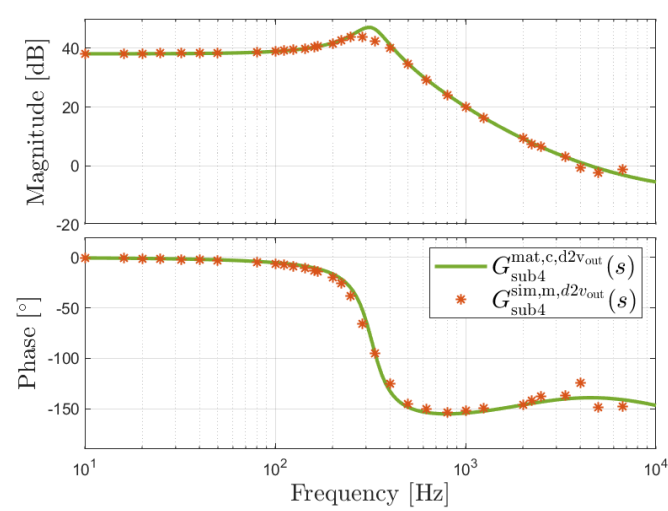

(b)

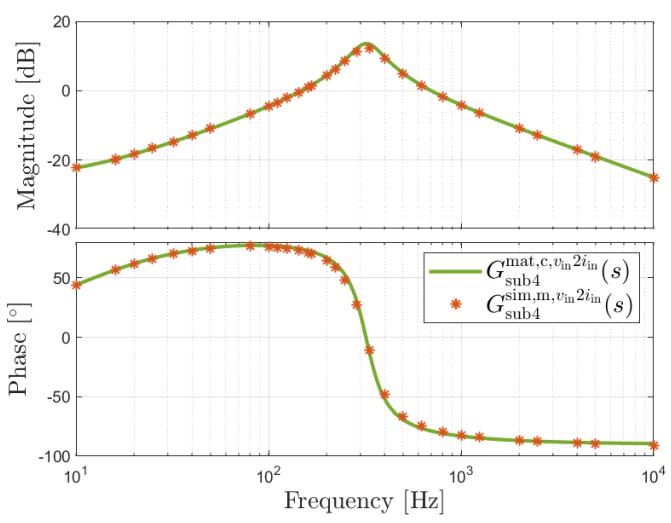

(d)

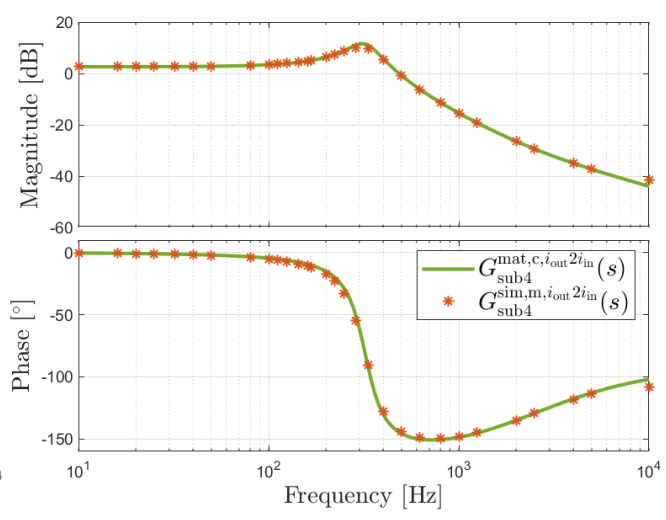

(f)

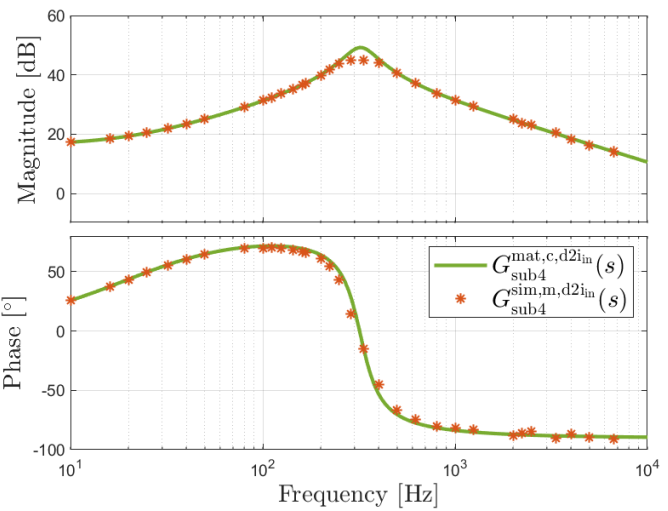

Figure 5. The Bode plots of the sub4 mathematically derived $G_{\text {sub4, }}^{\text {mat, }, 2 .}(s)$ and simulation measurement confirmed $G_{\text {sub4 }}^{\text {sim,m,2. }}(s)$ : (a) voltage transmittance $G_{\text {sub4, }}^{\text {mat, } \mathrm{v}_{\text {in }} 2 v_{\text {out }}}(s)$ according to Equations (1) and (13); (b) input admittance $G_{\text {sub4, }}^{\text {mat, } v_{\text {in }} 2 i_{\text {in }}}(s)$ according to Equations (2) and (14);

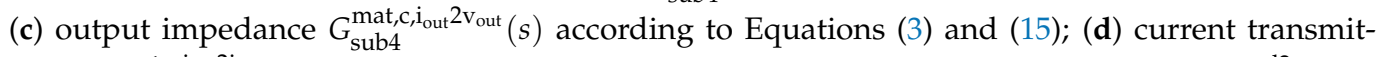
tance $G_{\text {sub4 }}^{\text {mat, }, i_{\text {out }} 2 i_{\text {in }}}(s)$ according to Equations (4) and (16); (e) control transmitance $G_{\text {sub4 }}^{\text {mat,c,d2 } v_{\text {out }}}(s)$ according to Equations (5) and (17); (f) control transmitance $G_{\text {sub4 }}^{\text {mat,c, d2i in }}$ (s) according to Equations (6) and (18).

\subsection{The sub1 and sub3 Passive Filters}

For the passive filters from sub1 and sub3 following four transfer functions (without the control dependencies) have been derived analogically to those from Section 3.1. As one 
can expect, results are the same for $s u b 1$ and $s u b 3$ and, therefore, for simplicity, the \# is used instead of 1 or 3 indexes:

$$
\begin{aligned}
& G_{\text {sub\# }}^{\text {mat, }, \text { vin } 2 v_{\text {out }}}(s)=\left.\frac{\widetilde{v}_{\text {sub\#, out }}(s)}{\widetilde{v}_{\text {sub\#, in }}(s)}\right|_{\tilde{i}_{\text {sub\#, out }}(s)=0}=\frac{s\left(r_{\mathrm{CF}} C_{\mathrm{F}}\right)+1}{s^{2}\left(L_{\mathrm{F}} C_{\mathrm{F}}\right)+s\left(C_{\mathrm{F}} r_{\mathrm{LF}}+C_{\mathrm{F}} r_{\mathrm{CF}}\right)+1} \\
& G_{\text {sub\# }}^{\text {mat, }, \text { in } 2 i_{\text {in }}}(s)=\left.\frac{\widetilde{i}_{\text {sub\#, in }}(s)}{\widetilde{v}_{\text {sub\#, in }}(s)}\right|_{\widetilde{i}_{\text {sub\#, out }}(s)=0}=\frac{s\left(C_{\mathrm{F}}\right)}{s^{2}\left(L_{\mathrm{F}} C_{\mathrm{F}}\right)+s\left(C_{\mathrm{F}} r_{\mathrm{LF}}+C_{\mathrm{F}} r_{\mathrm{CF}}\right)+1} \\
& G_{\text {sub\# }}^{\text {mat, }, \text { iout } 2 \mathrm{v}_{\text {out }}}(s)=\left.\frac{\widetilde{v}_{\text {sub\#,out }}(s)}{-\widetilde{i}_{\text {sub\#, out }}(s)}\right|_{\widetilde{v}_{\text {sub\#,in }}(s)=0}=\frac{s^{2}\left(C_{\mathrm{F}} L_{\mathrm{F}} r_{\mathrm{CF}}\right)+s\left(L_{\mathrm{F}}+C_{\mathrm{F}} r_{\mathrm{CF}} r_{\mathrm{LF}}\right)+1}{s^{2}\left(L_{\mathrm{F}} C_{\mathrm{F}}\right)+s\left(C_{\mathrm{F}} r_{\mathrm{LF}}+C_{\mathrm{F}} r_{\mathrm{CF}}\right)+1} \\
& G_{\text {sub\#, }}^{\text {mat, } \text { i }_{\text {out }} 2 i_{\text {in }}}(s)=\left.\frac{\widetilde{i}_{\text {sub\#,in }}(s)}{\widetilde{i}_{\text {sub\#, out }}(s)}\right|_{\widetilde{v}_{\text {sub\#,in }}(s)=0}=\frac{s\left(r_{\mathrm{CF}} C_{\mathrm{F}}\right)+1}{s^{2}\left(L_{\mathrm{F}} C_{\mathrm{F}}\right)+s\left(C_{\mathrm{F}} r_{\mathrm{LF}}+C_{\mathrm{F}} r_{\mathrm{CF}}\right)+1}
\end{aligned}
$$

where $G_{\text {sub\# }}^{\text {mat },, v_{\text {in }} 2 v_{\text {out }}}(s)$ describes relation between the output voltage to the input voltage; $G_{\text {sub\# }}^{\text {mat, }, v_{\text {in }} 2 \mathrm{i}_{\text {in }}}(s)$ is the input current to the input voltage; $G_{\text {sub\# }}^{\text {mat, }, i_{\text {out }} 2 v_{\text {out }}}(s)$ is the output voltage to the output current; $G_{\text {sub\# }}^{\text {mat, }, i_{\text {out }} 2 i_{\text {in }}}(s)$ is the input current to the output current. The Equation (22) is with minus just to match signs strategy used explained in Section 4.1. The Equations (19)-(22) with numeric values from Table 1 take the following shape:

$$
\begin{aligned}
& G_{\text {sub1 }}^{\text {mat }, c, v_{\text {in }} 2 v_{\text {out }}}(s)=\frac{1 \times 10^{-5} s+1}{1 \times 10^{-9} s^{2}+6 \times 10^{-5} s+1} \\
& G_{\text {sub1 }}^{\text {mat, }, v_{\text {in }} 2 \mathrm{i}_{\text {in }}}(s)=\frac{0.001 s}{1 \times 10^{-9} s^{2}+6 \times 10^{-5} s+1} \\
& G_{\text {sub1 } 1}^{\text {mat, } i_{\text {out }} 2 v_{\text {out }}}(s)=\frac{1 \times 10^{-11} s^{2}+1.5 \times 10^{-6} s+0.05}{1 \times 10^{-9} s^{2}+6 \times 10^{-5} s+1} \\
& G_{\text {sub1 }}^{\text {mat, }, i_{\text {out }} 2 \mathrm{i}_{\text {in }}}(s)=\frac{1 \times 10^{-5} s+1}{1 \times 10^{-9} s^{2}+6 \times 10^{-5} s+1}
\end{aligned}
$$

Similarly, sub3 can be written as:

$$
\begin{aligned}
& G_{\text {sub3 } 3,}^{\text {mat }, v_{\text {in }} 2 v_{\text {out }}}(s)=\frac{1 \times 10^{-6} s+1}{1 \times 10^{-9} s^{2}+6 \times 10^{-6} s+1} \\
& G_{\text {sub3 }}^{\text {mat, }, v_{\text {in }} 2 \mathrm{i}_{\text {in }}}(s)=\frac{0.0001 s}{1 \times 10^{-9} s^{2}+6 \times 10^{-6} s+1} \\
& G_{\text {sub3 }}^{\text {mat, }, i_{\text {out }} 2 v_{\text {out }}}(s)=\frac{1 \times 10^{-11} s^{2}+1.005 \times 10^{-5} s+0.05}{1 \times 10^{-9} s^{2}+6 \times 10^{-6} s+1} \\
& G_{\text {sub3 }}^{\text {mat, }, \text { i }_{\text {out }} 2 i_{\text {in }}}(s)=\frac{1 \times 10^{-6} s+1}{1 \times 10^{-9} s^{2}+6 \times 10^{-6} s+1}
\end{aligned}
$$

The Bode plots of the transfer functions represented by Equations (23)-(30) are shown in Figure 6. Their shapes are not optimised for fulfilment of particular clectro-magnetic compatibility requirements but just to be present in the analysis conducted in this research. A dedicated simulated verification of the sub-circuits was omitted here due to its complexity, but is included in the system-level simulations covered later in this paper. 
(a)

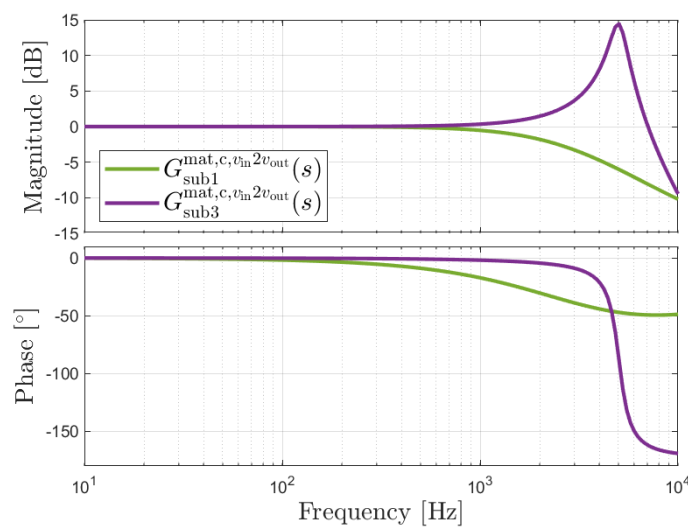

(c)

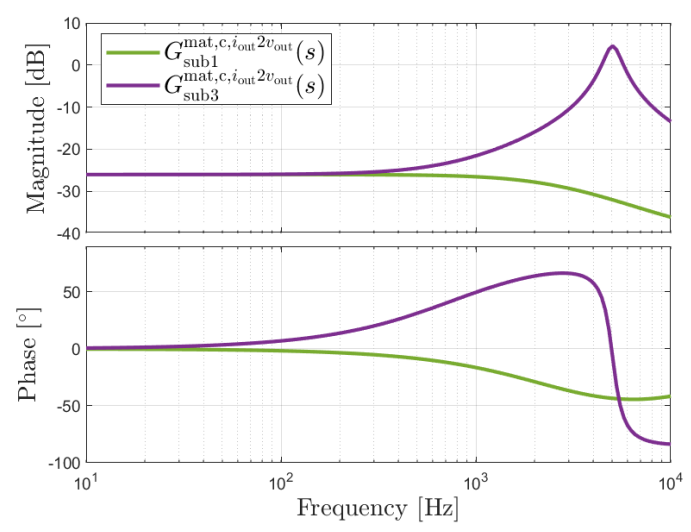

(b)

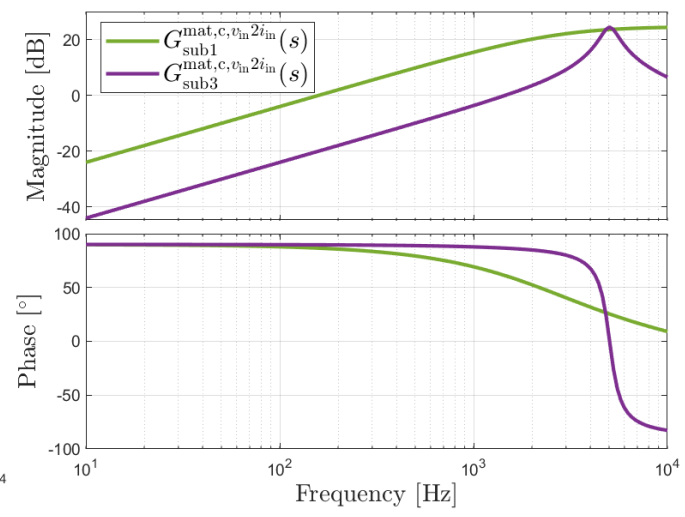

(d)

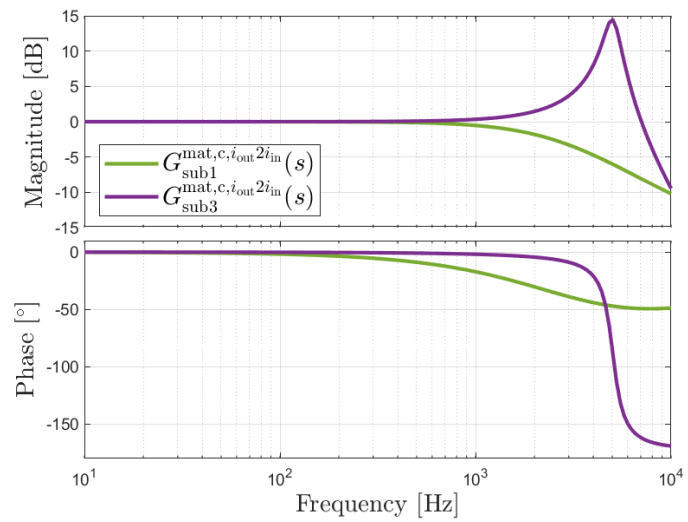

Figure 6. The Bode plots of the $s u b 1$ and $s u b 3$ passive $L C$ filters mathematically derived $G_{\text {sub\# }}^{\text {mat, }, 2 .}$. $(s)$ : (a) voltage transmittance $G_{\text {sub\# }}^{\text {mat, }, v_{\text {in }} 2 v_{\text {out }}}(s)$ according to Equations (19), (23) and (27); (b) input admittance $G_{\text {sub\# }}^{\text {mat, }, v_{\text {in }} 2 \mathrm{i}_{\text {in }}}(s)$ according to Equations (20), (24) and (28); (c) output impedance $G_{\text {sub\# }}^{\text {mat, }, \mathbf{i}_{\text {out }} 2 \mathrm{v}_{\text {out }}}(s)$ according to Equations (21), (25) and (29); (d) current transmittance $G_{\text {sub\# }}^{\text {mat, }, i_{\text {out }} 2 \mathrm{i}_{\text {in }}}$ (s) according to Equations (22), (26) and (30).

At this stage, a complete set of transfer functions describing sufficiently all of the four sub-systems is available. Therefore, system-level analyses can take place now.

\section{The System-Level Control Analysis}

The system-level control design must take into account interactions between PEC and surrounding them passive or active circuits. Therefore different analytical approach is needed when compared to the single PEC control design procedure described in [32]. The difference is located at stage of the input data preparation for the $\mathfrak{D}$-decomposition technique.

\subsection{Selected Aspects of the Two-Port Network Theory for the System-Level Analysis}

Foundation of the analysis relies on the well known two-port networks theory [33]. In this particular case we use modified inverse hybrid equation:

$$
\left[\begin{array}{c}
\widetilde{v}_{\text {sub\#,out }}(s) \\
\widetilde{i}_{\text {sub\#,in }}(s)
\end{array}\right]=\left[\begin{array}{cc}
G_{\text {sub\# }}^{\mathrm{v}_{\text {in }} 2 \mathrm{v}_{\text {out }}}(s) & G_{\text {sub\# }}^{\mathrm{i}_{\text {out }} 2 \mathrm{v}_{\text {out }}}(s) \\
G_{\text {sub\# }}^{\mathrm{v}_{\text {in }} 2_{\text {in }}}(s) & -G_{\text {sub\# }}^{\text {out }_{\text {oin }}}(s)
\end{array}\right]\left[\begin{array}{c}
\widetilde{v}_{\text {sub\#,in }}(s) \\
-\widetilde{i}_{\text {sub\#,out }}(s)
\end{array}\right]
$$

where, in case of not controlled sub-systems (passive circuits), the parameters matrix contains transfer functions represented by Equations (19)-(22) in general and in this particular case by Equations (23)-(26) for sub1 and by Equations (27)-(30) for sub3. The Equation (31) in form of a block diagram can be seen in Figure 7 . 


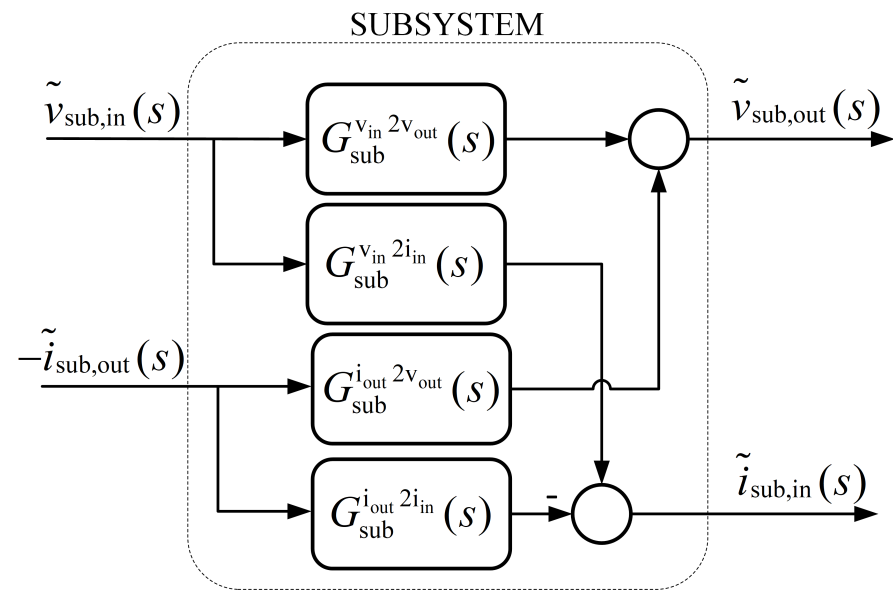

Figure 7. Block diagram of the two-port passive network described by Equation (31).

The passive two-port network shown in Figure 7 must be extended by additional functional blocks if it is meant to be used with a controlled sub-systems, see Figure 8 . The extension takes into account the closed-loop voltage control mechanism. Apart from the voltage controller $C^{\mathrm{v}}(s)$ and the plant control transfer functions, such as the control-tooutput voltage $G_{\text {sub }}^{\text {c2vot }}(s)$ and the control-to-input current $G_{\text {sub }}^{\text {c2i }}(s)$, it contains $G^{\mathrm{PWM}}(s)$, representing time delays driven by the Pulse Width Modulation (PWM) and $G^{\mathrm{A} 2 \mathrm{D}}(s)$, related to delays from the analog-to-digital (A2D) conversion.

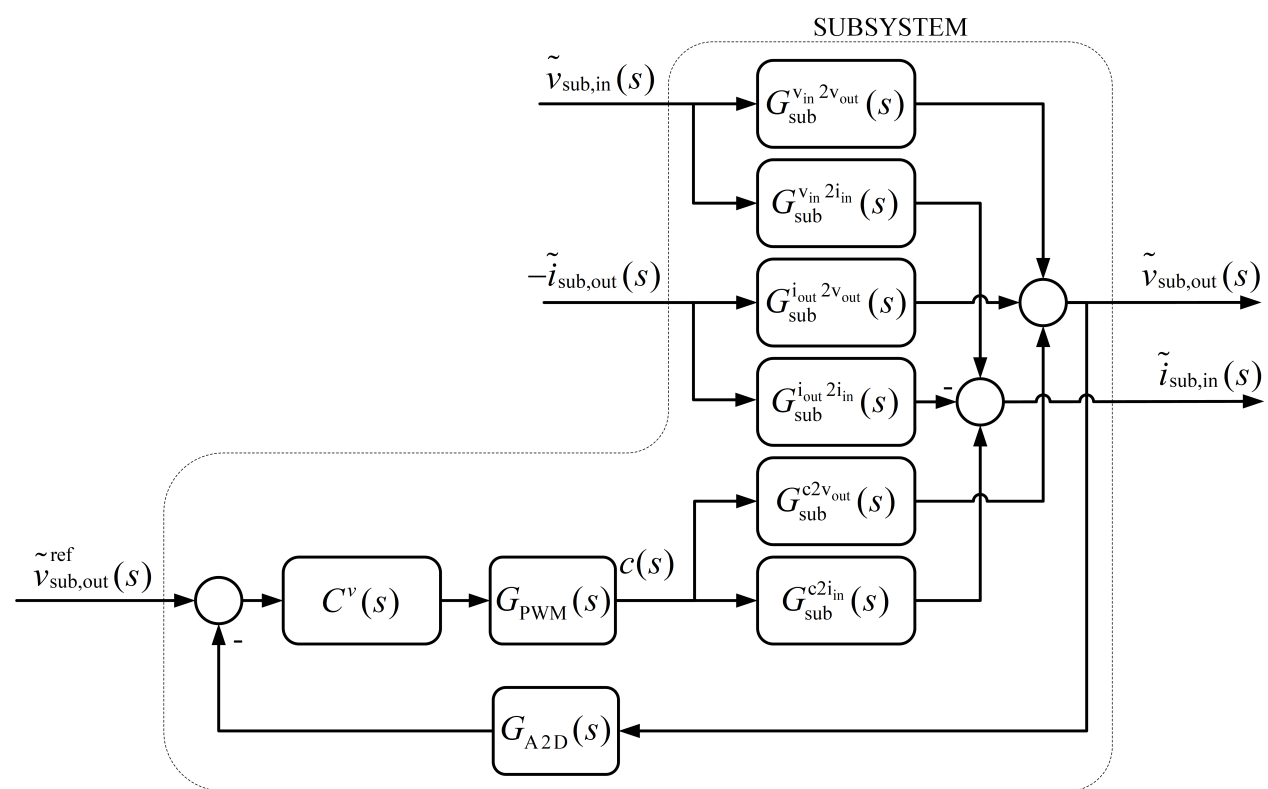

Figure 8. Block diagram of the two-port network taking into account a closed loop output voltage control loop with time delays coming from the PWM and the A2D conversion.

Dependencies shown in Figure 8 can be described by the following transfer functions:

$$
G_{\mathrm{CL}, \mathrm{v}, \text { sub }}^{\mathrm{v}_{\text {in }} 2 \mathrm{v}_{\text {out }}}(s)=\left.\frac{\widetilde{v}_{\text {sub }, \text { out }}(s)}{\widetilde{v}_{\text {sub,in }}(s)}\right|_{\widetilde{i}_{\text {sub }, \text { out }}(s), \widetilde{c}(s)=0}=\frac{G_{\text {sub }}^{\mathrm{v}_{\text {in }} 2 \mathrm{v}_{\text {out }}}(s)}{1+\left[C^{v}(s) G_{\mathrm{PWM}}(s) G_{\text {sub }}^{\mathrm{c} 2 \mathrm{v}_{\text {out }}}(s) G_{\mathrm{A} 2 \mathrm{D}}(s)\right]}
$$




$$
\begin{aligned}
& G_{\mathrm{CL}, \mathrm{v}, \text { sub }}^{\mathrm{v}_{\text {in }} 2 \mathrm{i}_{\text {in }}}(s)=\left.\frac{\widetilde{i}_{\text {sub,in }}(s)}{\widetilde{v}_{\text {sub,in }}(s)}\right|_{\widetilde{i}_{\text {sub,out }}(s), \widetilde{c}(s)=0}= \\
& G_{\mathrm{sub}}^{\mathrm{v}_{\text {in }} 2 \mathrm{i}_{\text {in }}}(s)-\frac{G_{\mathrm{sub}}^{\mathrm{v}_{\text {in }} 2 \mathrm{v}_{\text {out }}}(s) G_{\mathrm{sub}}^{\mathrm{c} 2 \mathrm{i}_{\text {in }}}(s) G_{\mathrm{A} 2 \mathrm{D}}(s) C^{v}(s) G_{\mathrm{PWM}}(s)}{1+\left[C^{v}(s) G_{\mathrm{PWM}}(s) G_{\mathrm{sub}}^{\mathrm{c} 2 \mathrm{v}_{\text {out }}}(s) G_{\mathrm{A} 2 \mathrm{D}}(s)\right]} \\
& G_{\mathrm{CL}, \mathrm{v}, \text { sub }}^{\mathrm{i}_{\text {out }} 2 \mathrm{v}_{\text {out }}}(s)=\left.\frac{\widetilde{v}_{\text {sub out }}(s)}{-\widetilde{i}_{\text {sub,out }}(s)}\right|_{\widetilde{v}_{\text {sub,in }}(s), \widetilde{c}(s)=0}=\frac{G_{\text {sub }}^{\mathrm{i}_{\text {out }} 2 \mathrm{v}_{\text {out }}}(s)}{1+\left[C^{v}(s) G_{\mathrm{PWM}}(s) G_{\mathrm{sub}}^{\mathrm{c} 2 \mathrm{v}_{\text {out }}}(s) G_{\mathrm{A} 2 \mathrm{D}}(s)\right]} \\
& G_{\mathrm{CL}, \mathrm{v}, \mathrm{sub}}^{\mathrm{i}_{\text {out }} 2 \mathrm{i}_{\text {in }}}(s)=\left.\frac{\widetilde{i}_{\text {sub,in }}(s)}{\widetilde{i}_{\text {sub,out }}(s)}\right|_{\widetilde{v}_{\text {sub }, \text { in }}(s), \widetilde{c}(s)=0}= \\
& G_{\mathrm{sub}}^{\mathrm{i}_{\text {out }} 2 \mathrm{v}_{\text {out }}}(s)+\frac{G_{\mathrm{sub}}^{\mathrm{i}_{\text {out } 2 \mathrm{v}_{\text {out }}}}(s) G_{\mathrm{sub}}^{\mathrm{c} 2 \mathrm{i}_{\text {in }}}(s) G_{\mathrm{A} 2 \mathrm{D}}(s) C^{v}(s) G_{\mathrm{PWM}}(s)}{1+\left[C^{v}(s) G_{\mathrm{PWM}}(s) G_{\mathrm{sub}}^{\mathrm{c} \mathrm{v}_{\text {out }}}(s) G_{\mathrm{A} 2 \mathrm{D}}(s)\right]}
\end{aligned}
$$

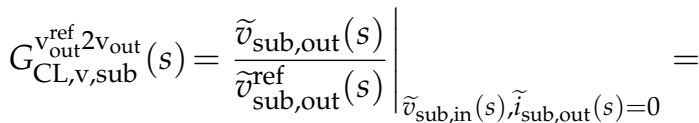

$$
\begin{aligned}
& \frac{C^{v}(s) G_{\mathrm{PWM}}(s) G_{\mathrm{sub}}^{\mathrm{c} 2 \mathrm{v}_{\text {out }}}(s)}{1+\left[C^{v}(s) G_{\mathrm{PWM}}(s) G_{\mathrm{sub}}^{\mathrm{c} \mathrm{v}_{\text {out }}}(s) G_{\mathrm{A} 2 \mathrm{D}}(s)\right]} \\
& G_{\mathrm{CL}, \mathrm{V}, \text { sub }}^{\mathrm{v} \text { ref } 2 \mathrm{i}_{\text {in }}}(s)=\left.\frac{\widetilde{i}_{\text {sub,in }}(s)}{\widetilde{v}_{\text {sub,out }}^{\text {ref }}(s)}\right|_{\widetilde{v}_{\text {sub,in }}(s), \widetilde{i}_{\text {sub,out }}(s)=0}= \\
& C^{v}(s) G_{P W M}(s) G_{\mathrm{s} 2}^{c 2} \mathrm{i}_{\text {in }}(s) \\
& 1+\left[C^{v}(s) G_{\mathrm{PWM}}(s) G_{\mathrm{sub}}^{\mathrm{c} 2 \mathrm{v}_{\text {out }}}(s) G_{\mathrm{A} 2 \mathrm{D}}(s)\right]
\end{aligned}
$$

The Equations (32)-(35) represent a set of equations associated with a two-port network containing closed-loop output voltage control. The Equations (36) and (37) are complementary ones taking into account the relations between perturbations in the voltage reference to the output voltage, Equation (36), and to the input current, Equation (37), of a single PEC. They are shown here for complete mathematical description of the Figure 8. In case of having a properly built simulation model, the $v_{\text {out }}^{\text {ref }} 2 v_{\text {out }}$ and $v_{\text {out }}^{\text {ref }} 2 i_{\text {in }}$ analyses can be conducted in the simulation and the equations are rather not necessary. The fact that the denominators are the same in all the Equations (32)-(37) should be emphasized. This means that it is sufficient to investigate just one of them as the characteristic equation to make a judgment on the overall stability of a sub-circuit under control.

\subsection{Mathematical Model of Two Systems Connected in Serial}

If we wish to conduct an analysis of the serial connection of the two-port networks, or rather two sub-systems, it is necessary to construct another block diagram, as shown in Figure 9. Here, we can see interactions between the two subsystems, e.g., sub1 and sub2, constituting a system, e.g., sys 1 from Figure 1. The output voltage and the current of the 1st sub-system are becoming the input voltage and the current of the 2nd sub-system. Of course the same is valid for the sys 2 consisting of the $s u b 3$ and the sub4. Further, the sys 3 from Figure 1 can be analysed analogically. 


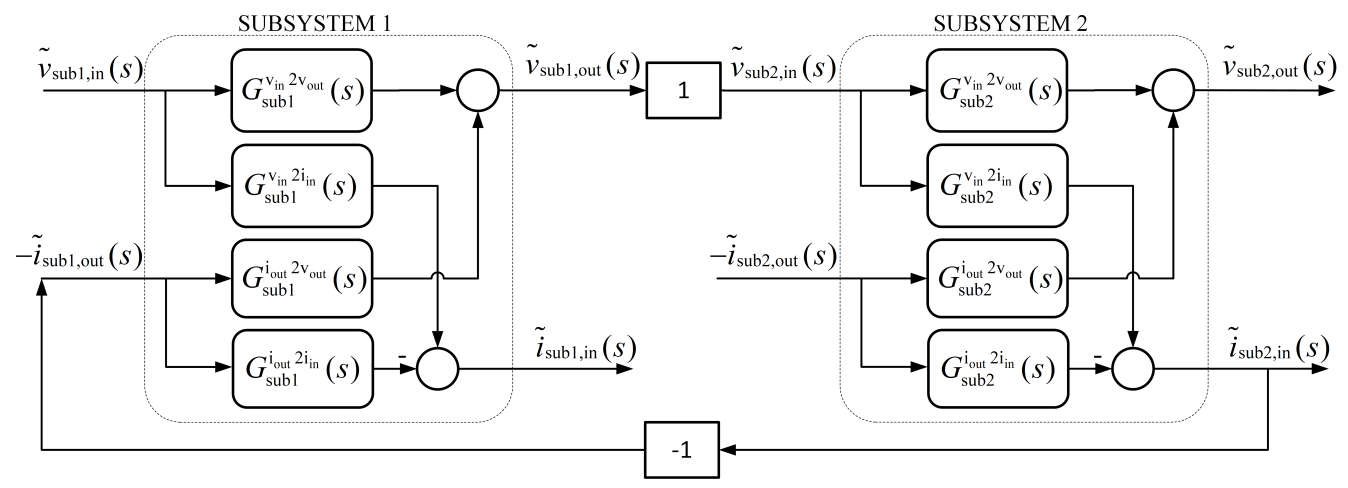

Figure 9. Block diagram of two subsystems connected in serial.

Relations, by means of the small signal perturbations, based on Figure 9 can be described by following formulas:

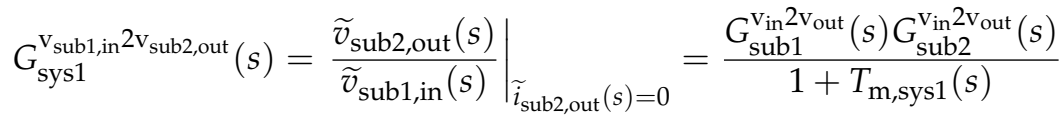

$$
\begin{aligned}
& G_{\text {sys1 }}^{\mathrm{v}_{\text {sun } 1 \text { in }} 2 \mathrm{i}_{\text {sub1,in }}}(s)=\left.\frac{\widetilde{i}_{\text {sub1,in }}(s)}{\widetilde{v}_{\text {sub1,in }}(s)}\right|_{\widetilde{i}_{\text {sub2,out }}(s)=0}=G_{\text {sub1 }}^{\mathrm{v}_{\text {in }} 2 \mathrm{i}_{\text {in }}}(s)+\frac{G_{\text {sub1 }}^{\mathrm{v}_{\text {in }} 2 \mathrm{v}_{\text {out }}}(s) G_{\text {sub2 }}^{\mathrm{v}_{\text {in }} 2 \mathrm{i}_{\text {in }}}(s) G_{\text {sub1 }}^{\mathrm{i}_{\text {out }} 2 \mathrm{i}_{\text {in }}}(s)}{1+T_{\mathrm{m}, \text { sys } 1}(s)} \\
& G_{\text {sys1 }}^{i_{\text {sub2,out }} 2 \mathrm{v}_{\text {sub2,out }}}(s)=\left.\frac{\widetilde{v}_{\text {sub2,out }}(s)}{-\widetilde{i}_{\text {sub2,out }}(s)}\right|_{\widetilde{v}_{\text {sub1,in }}(s)=0}= \\
& -G_{\text {sub2 }}^{\mathrm{i}_{\text {out }} 2 \mathrm{v}_{\text {out }}}(s)-\frac{G_{\text {sub2 }}^{\mathrm{i}_{\text {out }} 2 \mathrm{i}_{\text {in }}}(s) G_{\text {sub1 }}^{\mathrm{i}_{\text {out }} 2 \mathrm{v}_{\text {out }}}(s) G_{\text {sub2 }}^{\mathrm{v}_{\text {in }} 2 \mathrm{v}_{\text {out }}}(s)}{1+T_{\mathrm{m}, \text { sys } 1}(s)} \\
& G_{\text {sys1 }}^{i_{\text {sub1 }} 2 i_{\text {sub2,in }}}(s)=\left.\frac{\widetilde{i}_{\text {sub1,in }}(s)}{\widetilde{i}_{\text {sub2,out }}(s)}\right|_{\widetilde{v}_{\text {sub1,in }}(s)=0}=\frac{G_{\text {sub2 }}^{i_{\text {out }} 2 i_{\text {in }}}(s) G_{\text {sub1 }}^{i_{\text {out }} 2 i_{\text {in }}}(s)}{1+T_{\mathrm{m}, \text { sys1 }}(s)}
\end{aligned}
$$

where $T_{\mathrm{m}, \mathrm{sys} 1}(s)$ part of the characteristic equation can be written as:

$$
T_{\mathrm{m}, \mathrm{sys} 1}(s)=G_{\text {sub1 }}^{\mathrm{i}_{\text {out }} 2 \mathrm{v}_{\text {out }}}(s) G_{\mathrm{sub} 2}^{\mathrm{v}_{\text {in }} 2 \mathrm{i}_{\text {in }}}(s)
$$

Equation (42) depends on the input impedance of the sub1 and input admittance of the sub2. It stands for description of so called the minor loop. It means that in such a case it is sufficient to know only two transfer functions: (i) the $G_{\text {sub2 }}^{\mathrm{vin}_{\text {in }} 2 \mathrm{i}_{\text {in }}}(s)$ represented by Equation (33) in general or by Equation (2) in considered Boost converter case and (ii) the $G_{\text {sub1 }}^{\mathrm{i}_{\text {out }} 2 \mathrm{v}_{\text {out }}}(s)$ represented by Equation (34) in general or by Equation (3) in this case. Following a path of the general considerations, at level of the transmittances, the $T_{\mathrm{m} 1}$ can be written as:

$$
T_{\mathrm{m}, \mathrm{sys} 1}(s)=\frac{A_{1, \mathrm{sys} 1}(s)+C_{\mathrm{sys} 1}^{\mathrm{v}}(s) A_{2, \mathrm{sys} 1}(s)}{1+C_{\mathrm{sys} 1}^{\mathrm{v}}(s) A_{3, \mathrm{sys} 1}(s)}
$$

Taking into account the compensator transfer function at the system level:

$$
C_{\mathrm{sys} 1}^{\mathrm{v}}\left(s, K_{\mathrm{P}, \mathrm{sys} 1}, K_{\mathrm{I}, \mathrm{sys} 1}\right)=K_{\mathrm{P}, \mathrm{sys} 1}+\frac{K_{\mathrm{I}, \mathrm{sys} 1}}{s}
$$

it can be expressed as a function of the compensator gains:

$$
T_{\mathrm{m}, \mathrm{sys} 1}\left(s, K_{\mathrm{P}, \mathrm{sys} 1}, K_{\mathrm{I}, \mathrm{sys} 1}\right)=\frac{A_{1, \mathrm{sys} 1}(s)+C_{\mathrm{sys} 1}^{\mathrm{v}}\left(s, K_{\mathrm{P}, \mathrm{sys} 1}, K_{\mathrm{I}, \mathrm{sys} 1}\right) A_{2, \mathrm{sys} 1}(s)}{1+C_{\mathrm{sys} 1}^{\mathrm{v}}\left(s, K_{\mathrm{P}, \mathrm{sys} 1}, K_{\mathrm{I}, \mathrm{sys} 1}\right) A_{3, \mathrm{sys} 1}(s)}
$$


where:

$$
\begin{aligned}
& A_{1, \text { sys1 }}(s)=G_{\text {sub1 }}^{\mathrm{i}_{\text {out } 2 \mathrm{v}_{\text {out }}}(s)} G_{\mathrm{sub2}}^{\mathrm{v}_{\text {in }} 2 \mathrm{i}_{\text {in }}}(s)
\end{aligned}
$$

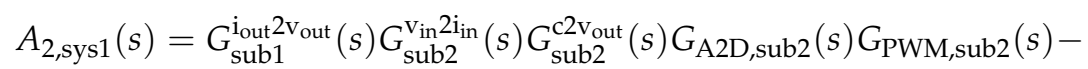

$$
\begin{aligned}
& G_{\text {sub1 }}^{\mathrm{i}_{\text {out } 2 \mathrm{v}_{\text {out }}}(s)} G_{\text {sub2 }}^{\mathrm{v}_{\text {in } 2 \mathrm{v}_{\text {out }}}}(s) G_{\mathrm{sub2}}^{\mathrm{c2i} \mathrm{i}_{\text {in }}}(s) G_{\mathrm{A} 2 \mathrm{D}, \text { sub2 }}(s) G_{\mathrm{PWM}, \text { sub2 }}(s) \\
& A_{3, \text { sys1 }}(s)=G_{\text {sub2 }}^{\text {c2 } v_{\text {out }}(s)} G_{\mathrm{A} 2 \mathrm{D}, \text { sub2 }}(s) G_{\mathrm{PWM}, \text { sub2 }}(s)
\end{aligned}
$$

Basing on Equations (45)-(48) and the values from Tables 1 and 2 following three sets

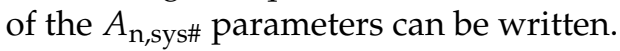

For the sys1:

$$
\begin{aligned}
& A_{1, \text { sys } 1}(s)=\frac{3533.5689045936397(112.5745806596871+s)\left(1+0.0000015 s+1 \times 10^{-11} s^{2}\right)}{\left(1+0.00006 s+1 \times 10^{-9} s^{2}\right)\left(3194470.798034441+718.7301418240824 s+s^{2}\right)} \\
& A_{2, \text { sys } 1}(s)=\frac{\mathrm{e}^{-0.0001 s}\left(8.6959 \times 10^{23}-6.7864 \times 10^{20} s-5.5933 \times 10^{16} s^{2}-2.7697 \times 10^{11} s^{3}-830915.2896 s^{4}-1.8779 s^{5}\right)}{\left(3194470.7980+718.7301 s+s^{2}\right)^{2}\left(9.9999 \times 10^{8}+60000 s+s^{2}\right)} \\
& A_{3, \text { sys } 1}(s)=-\frac{0.5494 \mathrm{e}^{-0.0001 s}(-27608.4982+s)(14184.3971+s)}{3194470.7980+718.7301 s+s^{2}}
\end{aligned}
$$

Each of the $A-$ parameters represents a set of mathematical operations which must be performed with certain precision in order to obtain satisfactory accuracy of the final solution. In case of some implementation on a digital signal processor, attention must be given to the precision of calculations. A priori to such implementation, an analysis of sensitivity must be conducted. In our case, this was not critical, since calculations were completed offline on a PC. We used 16 digits of precision, which has been proven to be sufficient.

For the sys2, the $T_{\mathrm{m}, \mathrm{sys} 2}(s)$ can be also calculated according to Equation (45) with modified indexes:

$$
T_{\mathrm{m}, \text { sys2 } 2}\left(s, K_{\mathrm{P}, \mathrm{sys} 2}, K_{\mathrm{I}, \mathrm{sys} 2}\right)=\frac{A_{1, \text { sys } 2}(s)+C_{\mathrm{sys} 2}^{\mathrm{v}}\left(s, K_{\mathrm{P}, \text { sys } 2}, K_{\mathrm{I}, \text { sys } 2}\right) A_{2, \mathrm{sys} 2}(s)}{1+C_{\mathrm{sys} 2}^{\mathrm{v}}\left(s, K_{\mathrm{P}, \mathrm{sys} 2}, K_{\mathrm{I}, \mathrm{sys} 2}\right) A_{3, \text { sys } 2}(s)}
$$

where:

$$
\begin{aligned}
& A_{1, \text { sys } 2}(s)=\frac{3533.5689(63.5501+s)\left(1+0.00001005 s+1 . \times 10^{-11} s^{2}\right)}{\left(1+0.000006 s+1 . \times 10^{-9} s^{2}\right)\left(4100892.0018+736.1119 s+s^{2}\right)} \\
& A_{2, \text { sys } 2}(s)=\frac{\mathrm{e}^{-0.0001 s}\left(2.5959 \times 10^{24}-8.1676 \times 10^{20} s-8.3803 \times 10^{16} s^{2}-9.7974 \times 10^{11} s^{3}-2903920.2424 s^{4}-2.1396 s^{5}\right)}{\left(4100892.0018+736.1119 s+s^{2}\right)^{2}\left(9.9999 \times 10^{8}+6000 s+s^{2}\right)} \\
& A_{3, \text { sys2 }}(s)=-\frac{0.5494 \mathrm{e}^{-0.0001 s}(-63759.6470+s)(14184.3971+s)}{4100892.0018+736.1119 s+s^{2}}
\end{aligned}
$$

Similarly, for the sys3, being serial connection of the sys 1 and the sys2, the $A_{\mathrm{n}, \mathrm{sys} 3}$ can be calculated and used for the $T_{\mathrm{m}, \mathrm{sys} 3}\left(s, K_{\mathrm{P}, \mathrm{sys} 3}, K_{\mathrm{I}, \mathrm{sys} 3}\right)$ calculation. They are not shown in this paper due to the complexity of the equations. Nevertheless, they have been easily managed in Matlab. Here, we assume that the gains $K_{\mathrm{P}, \mathrm{sys} 1}$ and $K_{\mathrm{I}, \mathrm{sys} 1}$ are fixed, and we are looking for new gains of the sys2. The gains may be different from their values suitable while operating as a single PEC not connected to a system, $K_{\mathrm{P}, \text { sys2 }}$ and $K_{\mathrm{I}, \mathrm{sys} 2}$. In such a way, it emulates situation, such as that seen in a fixed system where we cannot adjust settings. We can only adjust the settings of the PEC being connected.

Before proceeding, it is noteworthy that the $A_{n, \text { sys\# }}$ coefficients expressed by Equations (46)-(48) can be described in the frequency domain as a general complex number:

$$
A_{n, \text { sys\# }}(j \omega)=\operatorname{Re}\left[A_{n}(j \omega)\right]+j \operatorname{Im}\left[A_{n}(j \omega)\right]
$$

Such a representation aids an understanding $T_{\mathrm{m}, \text { sys\# }}\left(s, K_{\mathrm{P}, \text { sub\#\#},} K_{\mathrm{I}, \text { sub\# }}\right)^{\prime} \mathrm{s}$ contribution in the controller gains calculation formulas shown later in the paper. The obtained charac- 
teristic equation of two serial-connected sub-systems or systems can be further used for detailed analyses of the control solutions.

\section{Designation of Desired Output Voltage Compensator Gains Boundaries at the System Level by Means of the $\mathfrak{D}$-Decomposition Technique}

At this stage, the Neimark's $\mathfrak{D}$-decomposition technique [13] can be used with the characteristic Equations (45) and (49), and the $T_{\mathrm{m}, \mathrm{sys} 3}\left(s, K_{\mathrm{P}, \mathrm{sys} 3}, K_{\mathrm{I}, \mathrm{ssys} 3}\right)$ for the (GSB) calculation of each system. Moreover, the required control margins can be applied in the controllers gains selection process.

In order to implement such control margins at a system level, it is necessary to apply appropriate constraints, which may be different from those applicable in the case of a single PEC [18]. Nevertheless, in this article, the well-known and intuitive Gain Margin Phase Margin (GMPM) was used [34].

Calculation of the system-level gains $K_{\mathrm{P} \text {,sys\# }}$ and $K_{\mathrm{I} \text {,sys\# }}$ with desired control margins was taken into account and can be conducted according to the calculation path shown in Section 3 of [32] or [17,18]. It leads to the following functions:

$$
\begin{aligned}
& K_{\mathrm{P}, \mathrm{sys} \#}(\omega, a, b)=\frac{\operatorname{Im}\left[A_{1, \mathrm{sys} \#}(j \omega)\right]\left(-\operatorname{Im}\left[A_{2, \mathrm{sys}}(j \omega)\right]+a \operatorname{Im}\left[A_{3, \mathrm{sys}}(j \omega)\right]\right)-b^{2} \operatorname{Re}\left[A_{3, \text { sys\#}}(j \omega)\right]}{\operatorname{Den}} \\
& +\frac{-\left(a-\operatorname{Re}\left[A_{1, \mathrm{sys} \#}(j \omega)\right]\right)\left(-\operatorname{Re}\left[A_{2, \text { sys\# }}(j \omega)\right]+a \operatorname{Re}\left[A_{2, \mathrm{sys} \#}(j \omega)\right]\right)}{\operatorname{Den}} \\
& +\frac{b\left(\operatorname{Im}\left[A_{2, \mathrm{sys} \#}(j \omega)\right]-\operatorname{Im}\left[A_{3, \mathrm{sys} \#}(j \omega)\right] \operatorname{Re}\left[A_{1, \mathrm{sys} \#}(j \omega)\right]\right)}{\operatorname{Den}} \\
& +\frac{b\left(\operatorname{Im}\left[A_{1, \text { sys\# }}(j \omega)\right] \operatorname{Re}\left[A_{3, \text { sys\# }}(j \omega)\right]\right)}{\operatorname{Den}} \\
& K_{\mathrm{I}, \mathrm{sys} \#}(\omega, a, b)=-\frac{\omega\left(a^{2} \operatorname{Im}\left[A_{3, \mathrm{sys}}(j \omega)\right]+b^{2} \operatorname{Im}\left[A_{3, \mathrm{sys}}(j \omega)\right]+\operatorname{Im}\left[A_{2, \mathrm{sys}}(j \omega)\right] \operatorname{Re}\left[A_{1, \mathrm{sys} \#}(j \omega)\right]\right)}{\operatorname{Den}} \\
& +\frac{\omega\left(-a\left(\operatorname{Im}\left[A_{2, \text { sys\# }}(j \omega)\right]+\operatorname{Im}\left[A_{3, \text { sys\# }}(j \omega)\right] \operatorname{Re}\left[A_{1, \text { sys\# }}(j \omega)\right]-\operatorname{Im}\left[A_{1, \text { sys\# }}(j \omega)\right] \operatorname{Re}\left[A_{3, \text { sys\# }}(j \omega)\right]\right)\right)}{\operatorname{Den}} \\
& +\frac{\omega\left(b\left(-\operatorname{Im}\left[A_{1, \text { sys\# }}(j \omega)\right] \operatorname{Im}\left[A_{3, \text { sys\# }}(j \omega)\right]+\operatorname{Re}\left[A_{2, \text { sys\# }}(j \omega)\right]-\operatorname{Re}\left[A_{1, \text { sys\# }}(j \omega)\right] \operatorname{Re}\left[A_{3, \text { sys\# }}(j \omega)\right]\right)\right)}{\operatorname{Den}} \\
& +\frac{\omega\left(-\operatorname{Im}\left[A_{1, \text { sys\#}}(j \omega)\right] \operatorname{Re}\left[A_{2, \text { sys\# }}(j \omega)\right]\right)}{\operatorname{Den}} \\
& \text { where: }
\end{aligned}
$$

$$
\begin{aligned}
\text { Den } & =\operatorname{Im}\left[A_{2, \text { sys\# }}(j \omega)\right]^{2}+b^{2} \operatorname{Im}\left[A_{3, \text { sys\# }}(j \omega)\right]^{2}+2 b \operatorname{Im}\left[A_{3, \text { sys\# }}(j \omega)\right] \operatorname{Re}\left[A_{2, \text { sys\# }}(j \omega)\right] \\
& +\operatorname{Re}\left[A_{2, \text { sys\# }}(j \omega)\right]^{2}-2 a \operatorname{Re}\left[A_{2, \text { sys\# }}(j \omega)\right] \operatorname{Re}\left[A_{3, \text { sys\# }}(j \omega)\right]+b^{2} \operatorname{Re}\left[A_{3, \text { sys\# }}(j \omega)\right]^{2} \\
& -2 \operatorname{Im}\left[A_{2, \text { sys\# }}(j \omega)\right]\left(a \operatorname{Im}\left[A_{3, \text { sys\# }}(j \omega)\right]+b \operatorname{Re}\left[A_{3, \text { sys\# }}(j \omega)\right]\right) \\
& +a^{2}\left(\operatorname{Im}\left[A_{3, \text { sys\# }}(j \omega)\right]^{2}+\operatorname{Re}\left[A_{3, \text { sys\# }}(j \omega)\right]^{2}\right)
\end{aligned}
$$

Here, the $a$ and $b$ parameters can be understood as standing for the coordinates of an arbitrary point. The point is located in the complex plane where a polar plot type is located-this is the case when the Nyquist plot type is considered as a function of the pulsation $\omega$.By means of proper location of the arbitrary point (or points), we can shape the forbidden regions driven by the control requirements. In the case of searching for the GSB, the $a=-1$ and $b=0$ must be used. Of course such an assumption significantly simplifies Equations (51) and (52). In addition to the two equations, an expression for the $\Delta D_{0}$ hyperplane is required $[17,18,32]$. This is related to the characteristic equation by means of the real zero at the origin of the $s$-plane $(s=0)$. Its solution in this case leads to the following complementary criterion:

$$
\Delta D_{0} \Rightarrow K_{\mathrm{I}, \mathrm{sys} \#}(\omega, a, b)=0
$$


In short, it states that there is a cut off hyperplane at $K_{\mathrm{I}, \text { syst }}(\omega, a, b)=01 / V \cdot s$ and therefore it is independent of the $\omega$ and the $a$ and $b$.

\subsection{The Global Stability Boundary of the sys1}

The GSB of the sys1, based on the Equations (51)-(54), can be seen in Figure 10a. For comparison, there is also an equivalent Nyquist plot shown in Figure 10b. For simplicity, the Nyquistplots are for frequency range $\langle 0, \infty)$. Those remaining are simply mirror reflections. The advantage of the $\mathfrak{D}$-decomposition technique outcome becomes evident when results are compared. The results are clear. The grey area indicates the stable region with unambiguous indication of the border of stability depending on the gains values. The plots have three example gains sets marked by the colour dots. The dots are for different pulsations listed together with corresponding gains values in Table 3. The table also contains the other values used over the course of this paper.

(a)

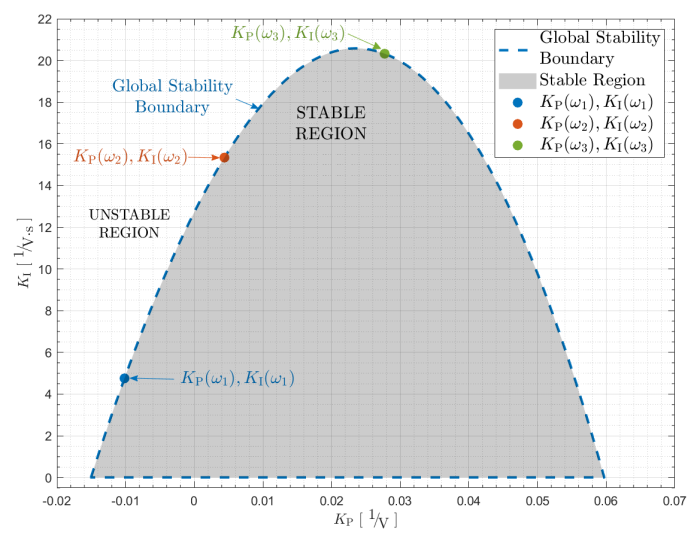

(b)

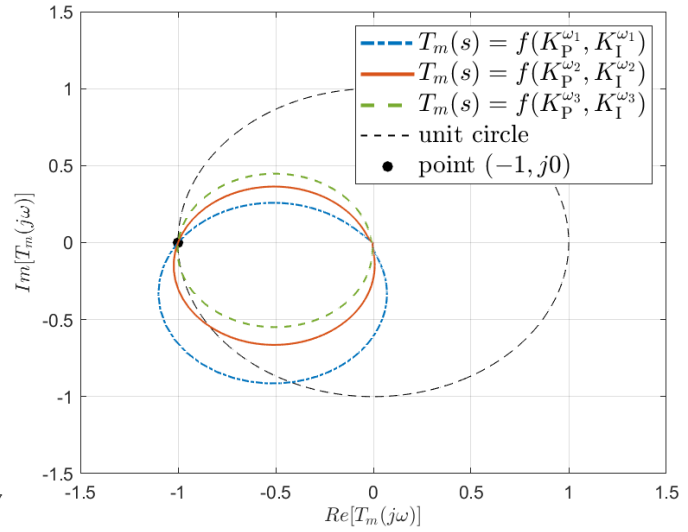

Figure 10. Visualisation of the sys1 (a) the global stability boundary (GSB) and (b) corresponding Nyquist plots with three PI-compensator gains sets as parameters. The GSB trajectories have been calculated according to Equations (51)-(54) with the $a=-1$ and the $b=0$. The gains (blue, red and green points) represent selected pulsations listed in Table 3.

Table 3. Values of the analysed gains $\left(K_{\mathrm{P}, \mathrm{sys} \#}, K_{\mathrm{I}, \text { sys }}\right)$ sets for sys1-sys3 shown in Figure 1 linked to the pulsations.

\begin{tabular}{|c|c|c|c|c|}
\hline System & $\begin{array}{l}\text { Pulsation } \\
\text { Designator }\end{array}$ & $\begin{array}{c}\text { Value of } K_{\mathbf{P}, \text { sys\# }} \\
{[1 / \mathrm{v}]}\end{array}$ & $\begin{array}{c}\text { Value of } K_{\mathrm{I}, \mathrm{sys} \#} \\
{[1 / \mathrm{v} \cdot \mathrm{s}]}\end{array}$ & $\begin{array}{c}\text { Value of Pulsation } \\
{[\mathrm{rad} / \mathrm{s}]}\end{array}$ \\
\hline \multirow{6}{*}{ sys 1} & $\omega_{1}$ & -0.01015 & 4.75360 & 990 \\
\hline & $\omega_{2}$ & 0.00440 & 15.34178 & 1990 \\
\hline & $\omega_{3}$ & 0.02773 & 20.32410 & 2990 \\
\hline & $\omega_{4}$ & -0.00644 & 4.72498 & 740 \\
\hline & $\omega_{5}$ & 0.00685 & 12.81386 & 2100 \\
\hline & $\omega_{6}$ & 0.02288 & 14.07938 & 2820 \\
\hline \multirow{3}{*}{ sys2 } & $\omega_{7}$ & -0.00526 & 3.51932 & 1180 \\
\hline & $\omega_{8}$ & 0.00723 & 6.39331 & 2160 \\
\hline & $\omega_{9}$ & 0.01942 & 3.49777 & 2860 \\
\hline \multirow{3}{*}{ sys3 } & $\omega_{10}$ & -0.010133 & 0.50578 & 342 \\
\hline & $\omega_{11}$ & -0.004123 & 1.15311 & 662 \\
\hline & $\omega_{12}$ & 0.002443 & 0.50923 & 876 \\
\hline
\end{tabular}

Based on the three selected gains sets for $\omega_{1} . . \omega_{3}$, the voltages $v_{\text {sub1,out }}^{\text {mat, }}$ and $v_{\text {sub2,out }}^{\text {mat,c }}=$ $v_{\text {sys1,out }}^{\text {mat, }}$ in the sys 1 have been calculated, see Figure 11. They are calculated with constant resistive load $R_{\text {out }}=33.3 \Omega$. Here, the sys 1 input voltage was also at three different levels, $v_{\text {sub1,in }}^{\text {ref }}=v_{\text {sys1,in }}^{\text {ref }}$. It reflects an acceptable tolerance of the rated input voltage of sub2, 
$V_{\text {sub2,in }}^{\text {rtd }}=30 \mathrm{~V} \pm 10 \%$, with negligible voltage droop across the $r_{\mathrm{LF}}$. The voltage at the filter output, $v_{\text {sub1,out }}^{\text {mat, }}$, shown in Figure 11a, stands for the input voltage of the sub2. The sys1 output voltage can be seen in Figure 11b. The obtained results demonstrate undamped oscillations at the three different frequencies according to the pulsations $\omega_{1} . . \omega_{3}$. Such results clearly indicate operation of the system at the GSB. Different sine-wave amplitudes at different input voltages for the same set of gains are related to the operating points at the constant load resistance. It can be noted that the sine-wave amplitudes are significantly different for each set of gains. The smallest is for the $\omega_{3}$, which is the highest pulsation and, therefore, is under the strongest low-pass filtering effect shown in Figures 5 and 6.

The same GSB calculation procedure is applicable to the sys 2 and sys3, although we are more interested in gains which can guarantee particular dynamic properties.

(a)

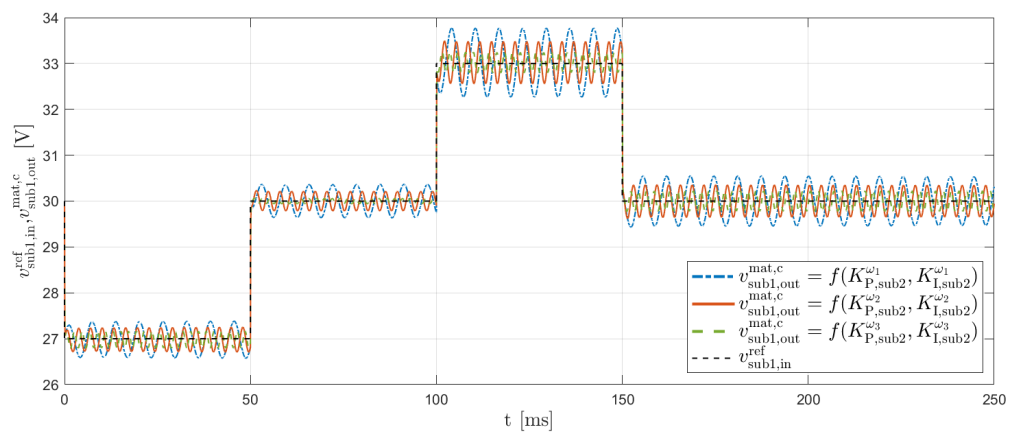

(b)

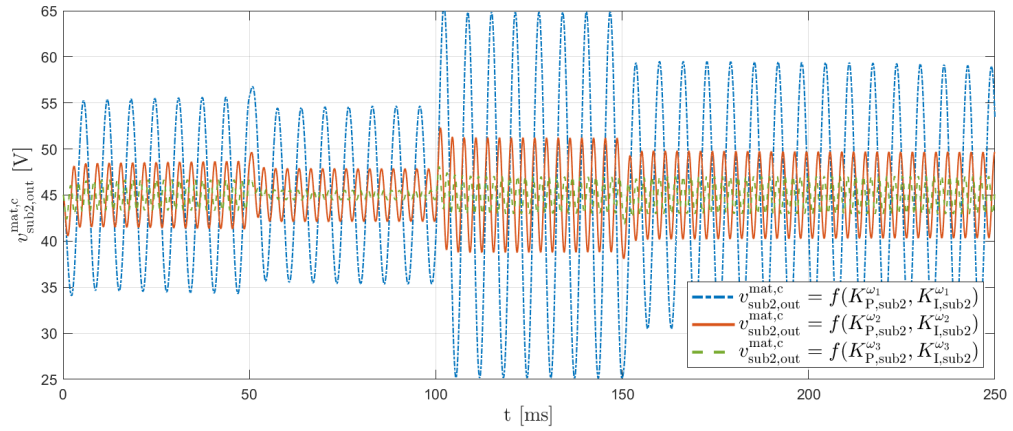

Figure 11. Mathematically calculated voltages in the sys 1 with the sub2 PI compensator gains at pulsations $\omega_{1} . . \omega_{3}$ according to Table 3, located at the GSB shown in Figure 10: (a) the sys1 input voltage, $v_{\text {sys1,in }}^{\text {ref }}=v_{\text {sub1,in }}^{\text {ref }} \approx V_{\text {sub2,in }}^{\text {rtd }}=30 \mathrm{~V} \pm 10 \%$, and the output voltage $v_{\text {sub1,out }}^{\text {mat, }}(\mathbf{b})$ the sys1 output voltage $v_{\text {sys1,out }}^{\text {mat,c }}=v_{\text {sub2,out }}^{\text {mat, }}$ stabilized at $45 \mathrm{~V}$. The results are for constant resistive load, $R_{\text {out }}=33.3 \Omega$, corresponding to the output power of $108 \mathrm{~W}$.

\subsection{Systam-Level Boundaries Driven by the GMPM Criterion}

The gains boundaries calculated by means of the $\mathfrak{D}$-decomposition technique according to the GMPM criterion can be seen in Figure 12. For the sys1, the Gain and the Phase margins were $20 \mathrm{~dB}$ and $70^{\circ}$, respectively. The calculated gains region (green colour) within the GSB (grey colour) can bee seen in Figure 12a. The corresponding Nyquist plot is shown in Figure 12b. Here, similarly to the data shown in Figure 10, three different pulsations are considered, $\omega_{4} . . \omega_{6}$ according to Table 3 .

The same is shown for the sys 2 and the sys 3 in Figure 12c,d and Figure 12e,f, respectively. In the case of sys2, the Gain and Phase margins were $12.5 \mathrm{~dB}$ and $80^{\circ}$, respectively, and the gains points are at $\omega_{7} . . \omega_{9}$ from Table 3 . The sys 3 margins were $12.5 \mathrm{~dB}$ and $17.5^{\circ}$ and the gains points located at $\omega_{10} . . \omega_{12}$ from Table 3 .

A reduction of the margins was driven by the availability of the solutions. This can be visually recognized while comparing the green regions. As expected, the smallest one is for 
the sys 3 case. The reduction between the sys 1 and the sys 2 operating independently comes from the fact that they are the same circuits working at different duty cycle steady-state operating points.

(a)

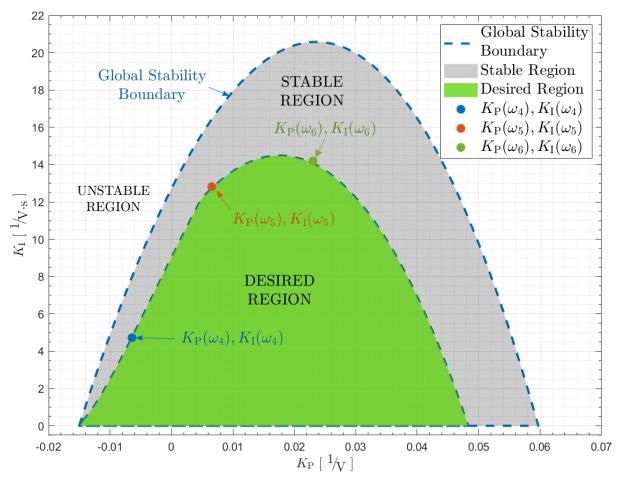

(c)

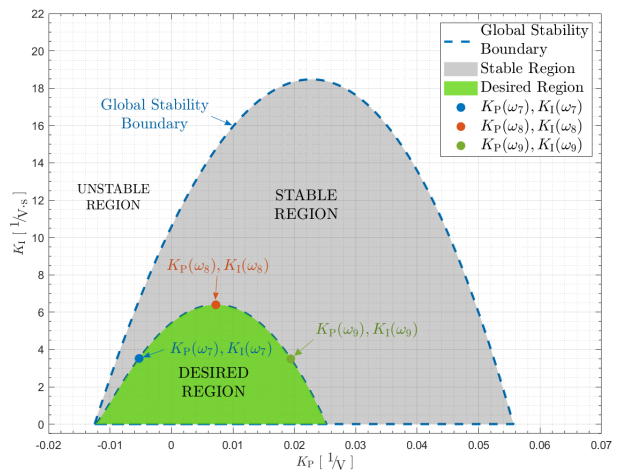

(e)

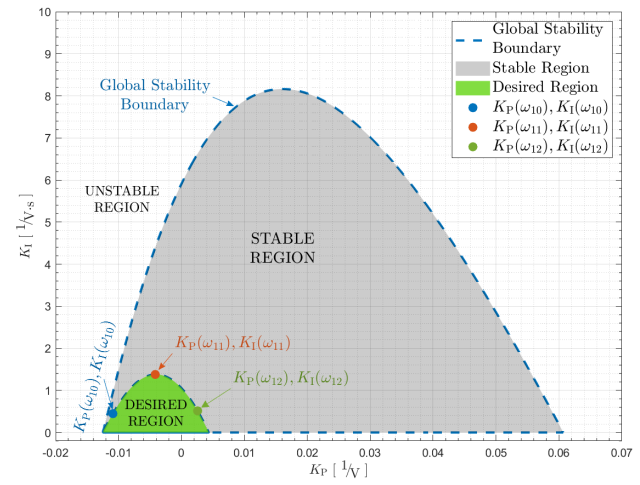

(b)

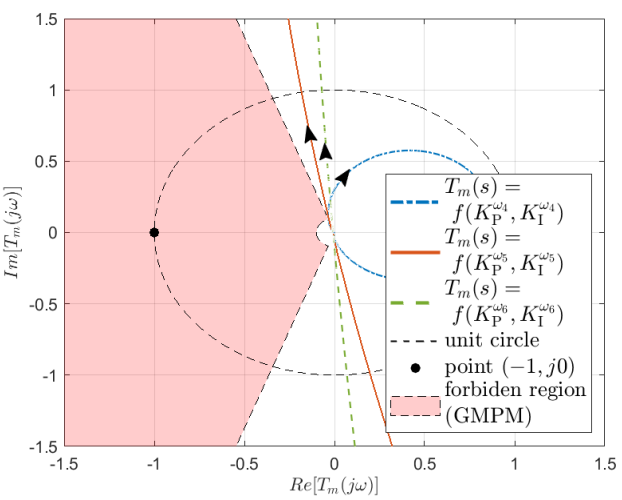

(d)

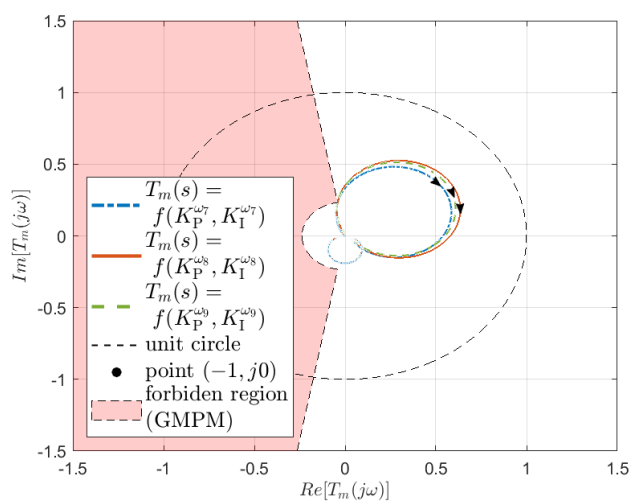

$(\mathbf{f})$

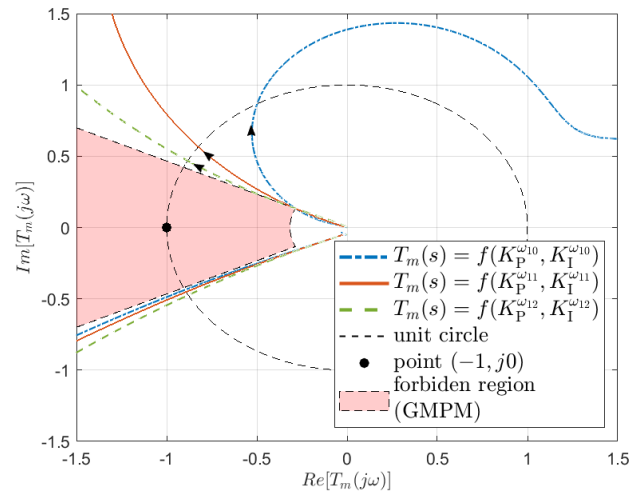

Figure 12. Visualisation of the $K_{\mathrm{P}, \mathrm{sys} \#}$ and $K_{\mathrm{I}, \mathrm{sys} \#}$ gains regions calculated by means of the $\mathfrak{D}$ decomposition technique according to Equations (51)-(54) for systems shown in Figure 1: (a) the sys $1 ;$ (c) the sys2; (e) the sys3. The corresponding Nyquist plots with forbidden regions according to the GMPM criterion can be seen in subplots $(\mathbf{b}, \mathbf{d}, \mathbf{f})$, respectively. The gains (blue, red and green points) represent selected pulsations, $\omega_{4}-\omega_{12}$, listed in Table 3.

\subsection{Mathematical Calculation Results for the sys1, sys2 and sys3}

All the gains values calculated according to the pulsations $\omega_{4} . . \omega_{12}$ are listed in Table 3. The corresponding results for sys 1 and sys 2 working independently can be seen in Figure 13. They are calculated mathematically. 
In the case of sys1, the gains at the highest pulsation $\omega_{6}$ provide the best performance in terms of the output voltage overshoot, $6.4 \%$, and the settling time, $7.89 \mathrm{~ms}$, Figure 13b. Therefore, these gains are used as fixed values during the $s y s 3$ analysis, where sys 2 gains are adjusted.

In case of the sys 2 , the smallest output voltage overshoot, $4.6 \%$, is offered by gains at $\omega_{9}$, although the shortest settling time, $5.18 \mathrm{~ms}$, belongs to $\omega_{8}$, see Figure $13 \mathrm{~d}$.

The situation appears to change when the sys 3 is considered, see Figure 14. If we use the gains of sys 1 according to the $\omega_{6}$ and $\omega_{7} . . \omega_{9}$ for sys 2 (selected for independent operation), the obtained results are unacceptable, see Figure $14 \mathrm{a}, \mathrm{b}$. The results are to be compared with Figure 13b,d, respectively. Therefore, new sys3 gains have been selected according to guidelines from Figure 12e. The obtained voltages can be seen in Figure 14c, d. Here, one can see significant improvements. The smallest output voltage overshoot, $4.0 \%$ in $v_{\text {sub2,out }}^{\text {mat, }}$ and $4.5 \%$ in $v_{\text {sub4,out }}^{\text {mat, }}$, is offered by gains at $\omega_{12}$. The settling times are $2.83 \mathrm{~ms}$ and $2.88 \mathrm{~ms}$, respectively. The $\omega_{12}=876 \mathrm{rad} / \mathrm{s}$ is the highest pulsation and this, in such particular system configuration, could be considered as a gains selection guideline. Nevertheless, further research is to be conducted before such a guideline can be generalized.

(a)

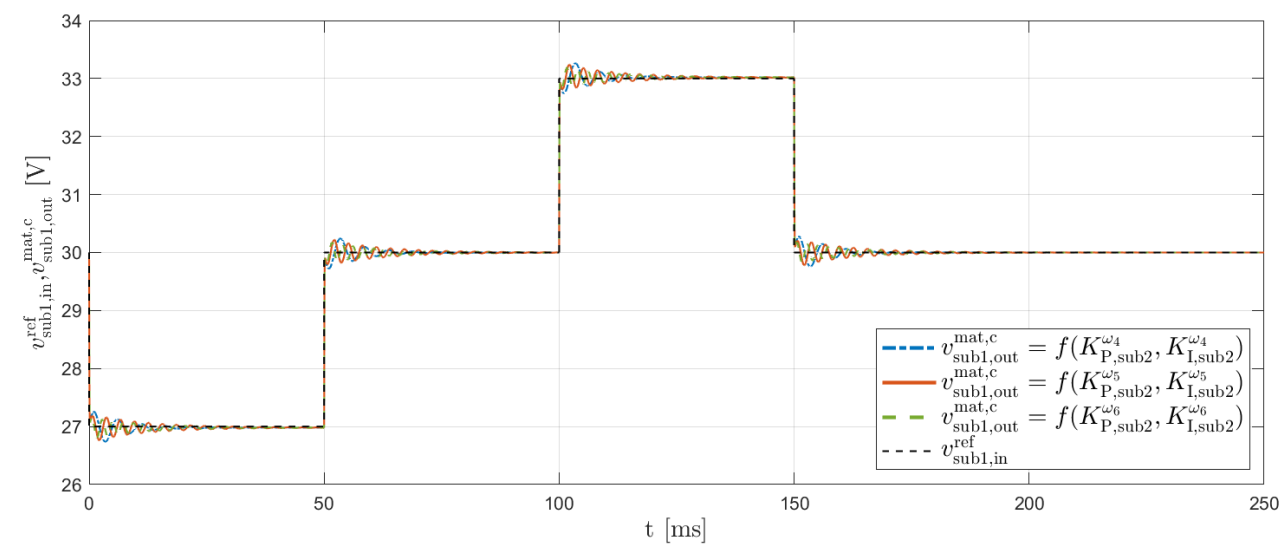

(b)

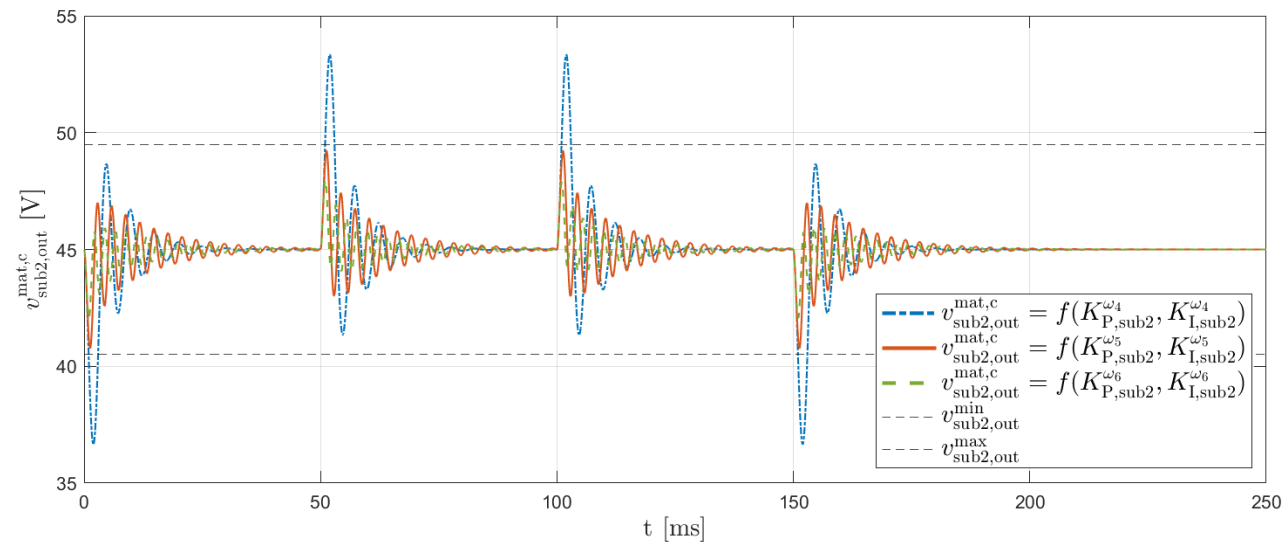

Figure 13. Cont. 
(c)

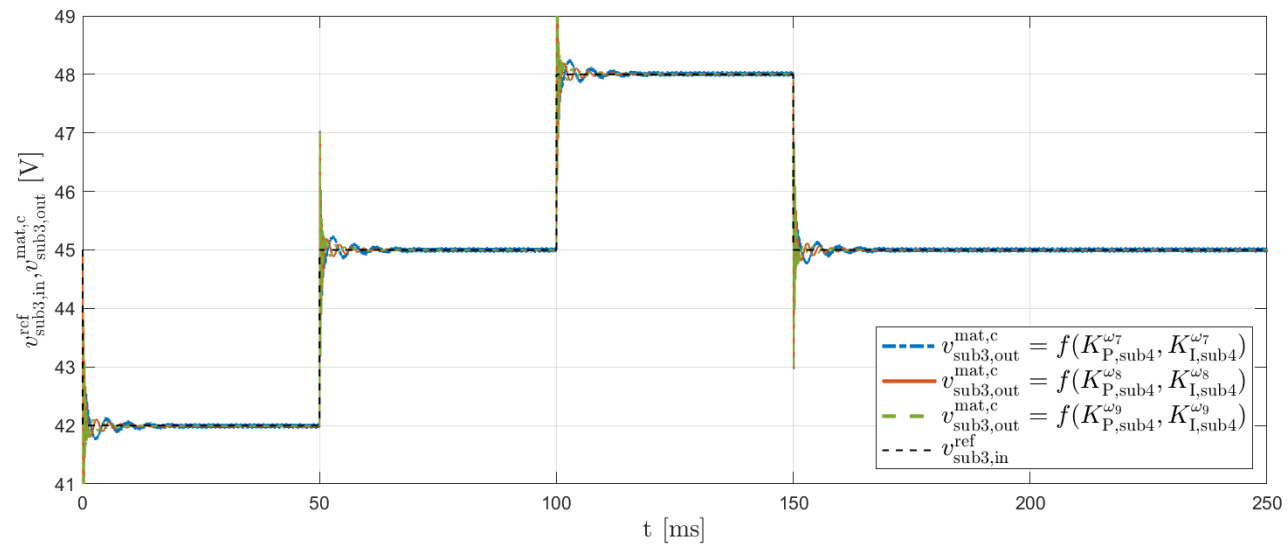

(d)

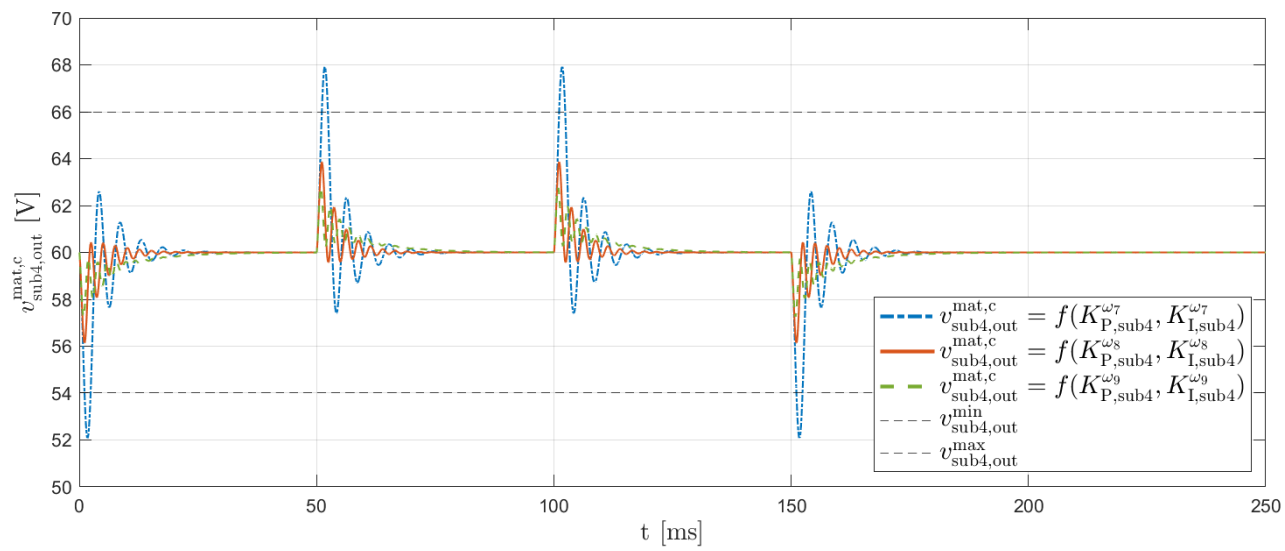

Figure 13. Mathematically calculated voltages in working independently sys1 and sys2: (a) sub1 of sys1 input voltage, $v_{\text {sys1,in }}^{\text {ref }}=v_{\text {sub1,in }}^{\text {ref }} \approx V_{\text {sub2,in }}^{\text {rtd }}=30 \mathrm{~V} \pm 10 \%$ and output voltage, $v_{\text {sub1,out }}^{\text {mat,c }}$ (b) $s u b 2$ of sys 1 output voltage, $v_{\mathrm{sys} 1, \mathrm{out}}^{\mathrm{mat}, \mathrm{c}}=v_{\mathrm{sub2, \text {out }}}^{\text {mat, }}$ stabilized at $45 \mathrm{~V}$; (c) sub3 of sys2 input voltage, $v_{\text {sys2,in }}^{\text {ref }}=v_{\text {sub3,in }}^{\text {ref }} \approx V_{\text {sub4,in }}^{\text {rtd }}=45 \mathrm{~V} \pm 10 \%$; (d) sub4 of sys2 output voltage, $v_{\text {sys2,out }}^{\text {mat,c }}=v_{\text {sub4,out }}^{\text {mat,c }}$ stabilized at $60 \mathrm{~V}$. The results are with the PI compensator gains at pulsations $\omega_{4} . . \omega_{9}$ according to Table 3. They are located in the $K_{\mathrm{P}}$ and $K_{\mathrm{I}}$ coordinates to satisfy the tuning criterions shown in Figure $12 \mathrm{a}$,c. The results are for constant resistive load, $R_{\text {out }}=33.3 \Omega$, corresponding to the output power of $108 \mathrm{~W}$. They are calculated in accordance to the control structure shown in Figure 2.

(a)

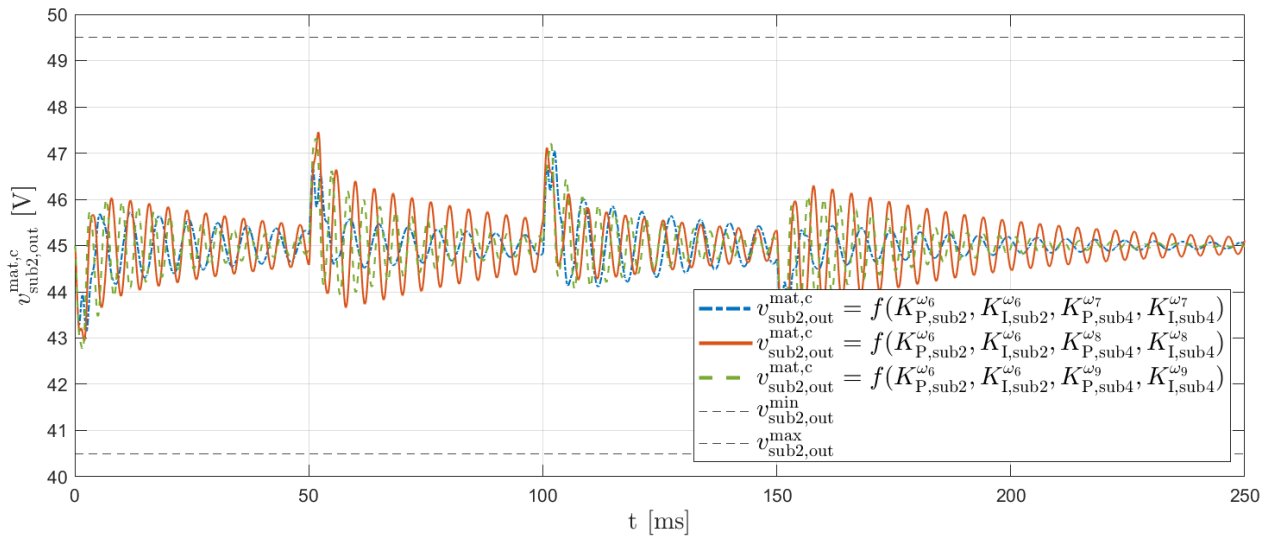

Figure 14. Cont. 
(b)

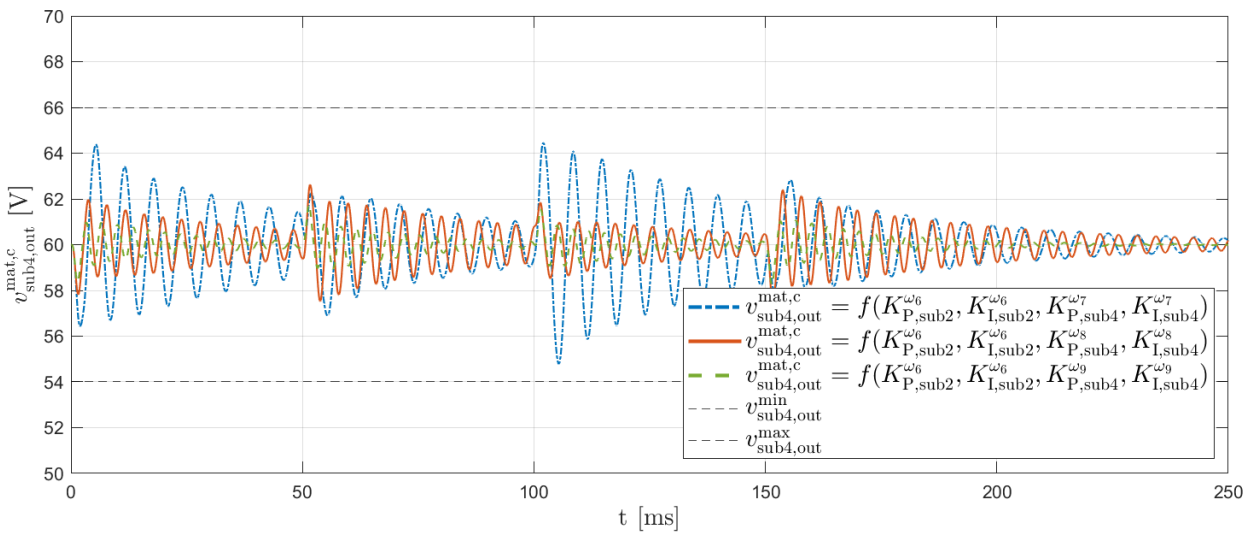

(c)

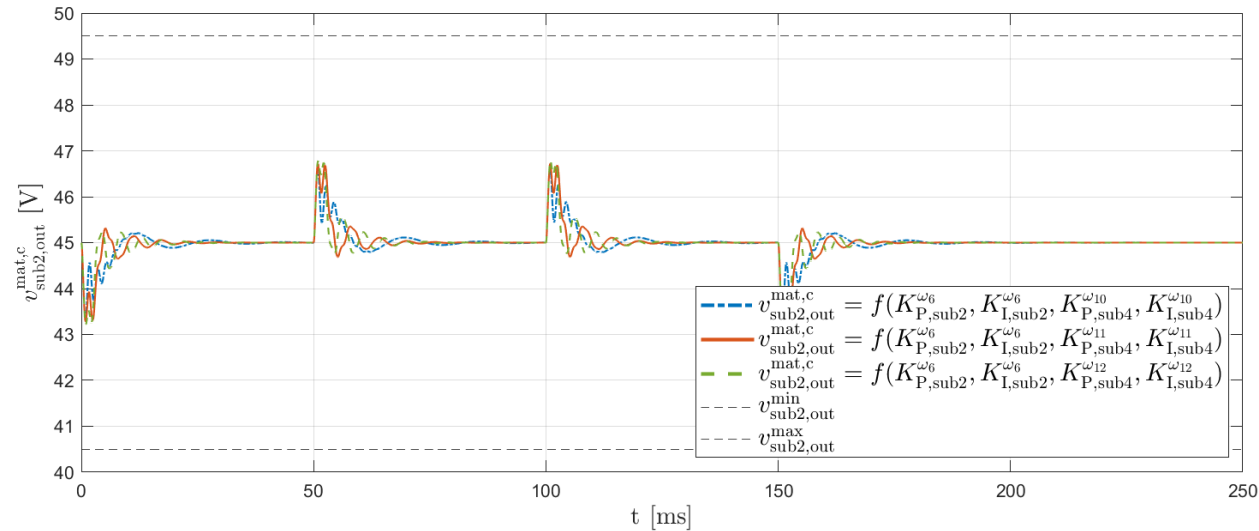

(d)

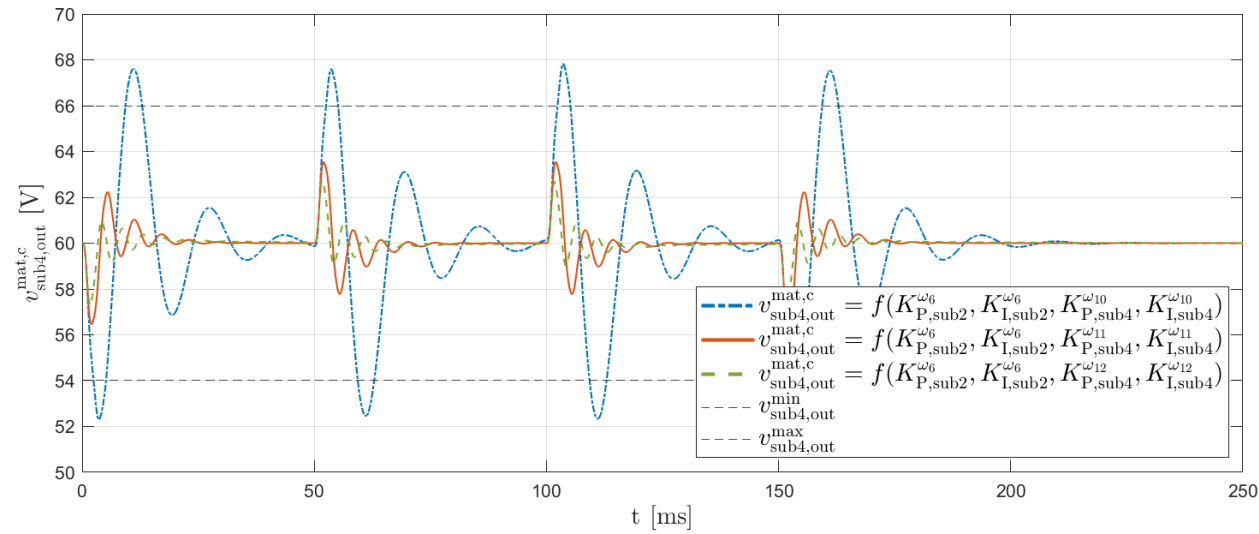

Figure 14. Mathematically calculated voltages in sys3 shown in Figure 1 with sub2 regulator gains at the $\omega_{6}$ from Table 3 and sub4 gains: $(\mathbf{a}, \mathbf{b})$ not optimised - the same as in the case of independently operating sys 2 at $\omega_{7} . . \omega_{9} ;(\mathbf{c}, \mathbf{d})$ optimised-according to the $K_{\mathrm{P}}$ and $K_{\mathrm{I}}$ located at $\omega_{10} . . \omega_{12}$ to satisfy the tuning criteria shown in Figure 12e. The results are for sys 3 constant resistive load, $R_{\text {out }}=33.3 \Omega$, corresponding to the output power of $108 \mathrm{~W}$. They are calculated in accordance to the output voltage, $v_{\text {sys3,out }}^{\text {mat, }}=v_{\text {sub4,out }}^{\text {mat, }}$, control structure shown in Figure 2. The input voltage profile, $v_{\text {sys3, in }}^{\text {mat,c }}=v_{\text {sub1,in }}^{\text {mat, }}$ was the same as that shown in Figure 13a.

\subsection{Simulation Results for the sys3}

The mathematical results presented in the previous section have been confirmed in a simulation model. The model done in Simulink has been verified experimentally [32]. Apart from the circuit components' equivalent series resistances, this approachtakes into account non-linear effects of the switches with their $V_{D S}$ characteristics according to the components data sheet. It is also equipped with saturations of the controllers, $0-65 \%$. The 
obtained results shown in Figure 15 (can be compared with Figure 14) confirm correctness of the mathematical modelling in a qualitative way when looking at the amplitudes. A good quantitative match can be seen for frequencies, especially when compensators operate in the linear range without saturation. The subtle differences in models contribute to less dumping in the simulation, although the overall dynamics picture is well preserved. The corresponding currents of the sys 3 can be seen in Figure 16. Analysing these currents aids our understanding of the processes occurring PES during transients.

In both models, mathematical and simulation, the $\mathfrak{D}$-decomposition technique merged with the GMPM criterion stands for an intuitive and precise means for control design of relatively small PES. The large PES approach, based on the mathematical system representation, may be a more efficient method from a computing time point of view.

(a)

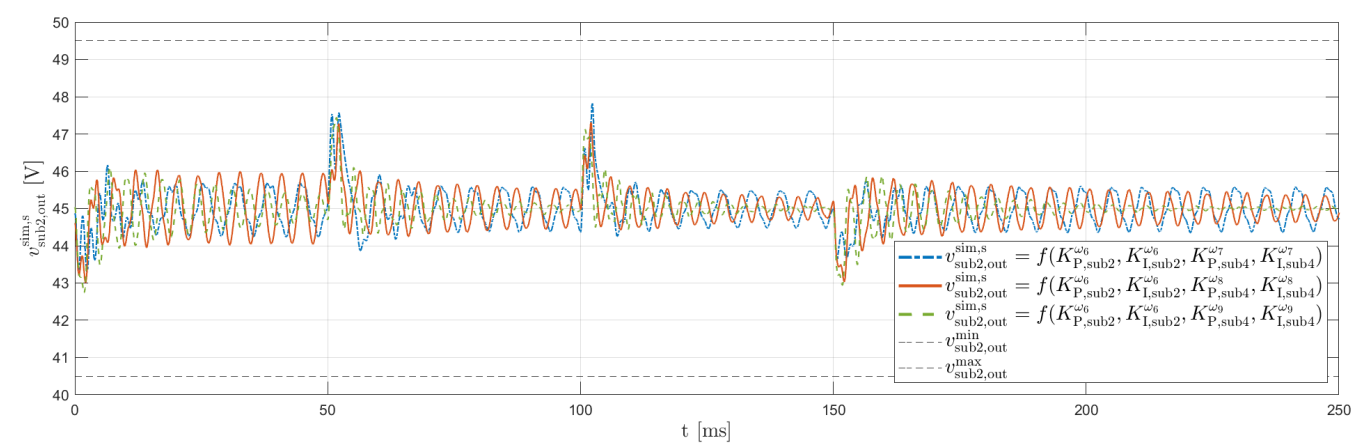

(b)

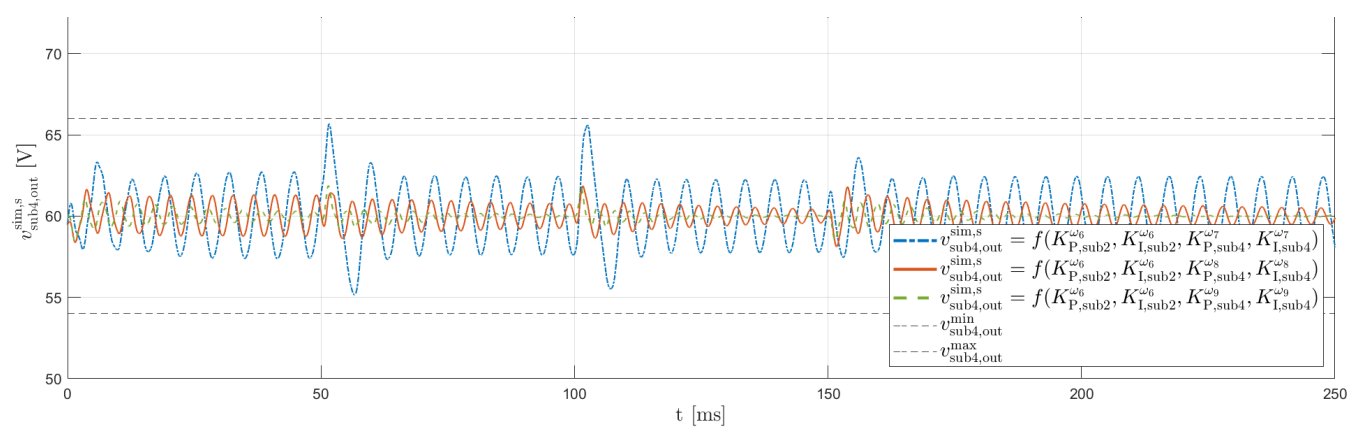

(c)

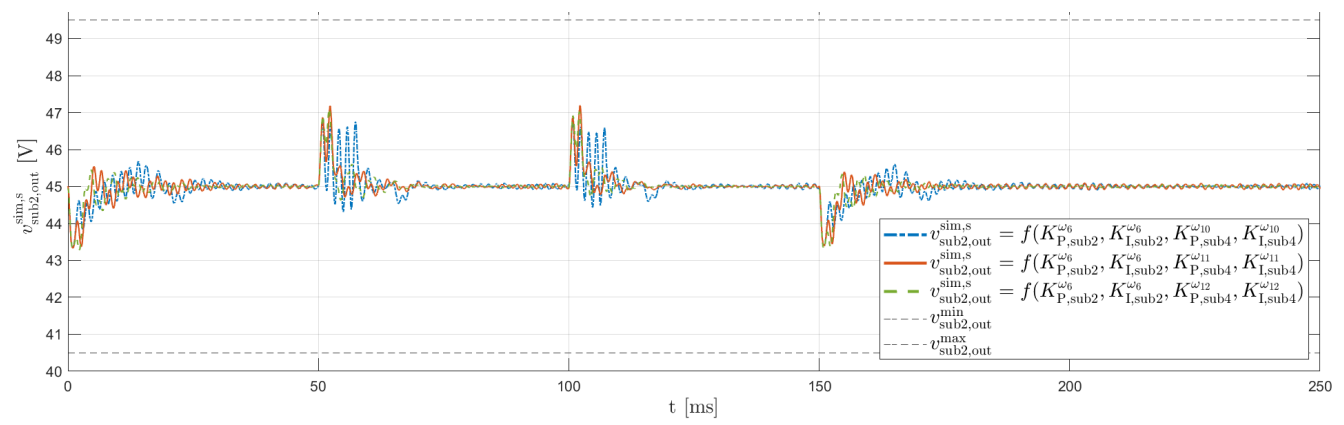

Figure 15. Cont. 
(d)

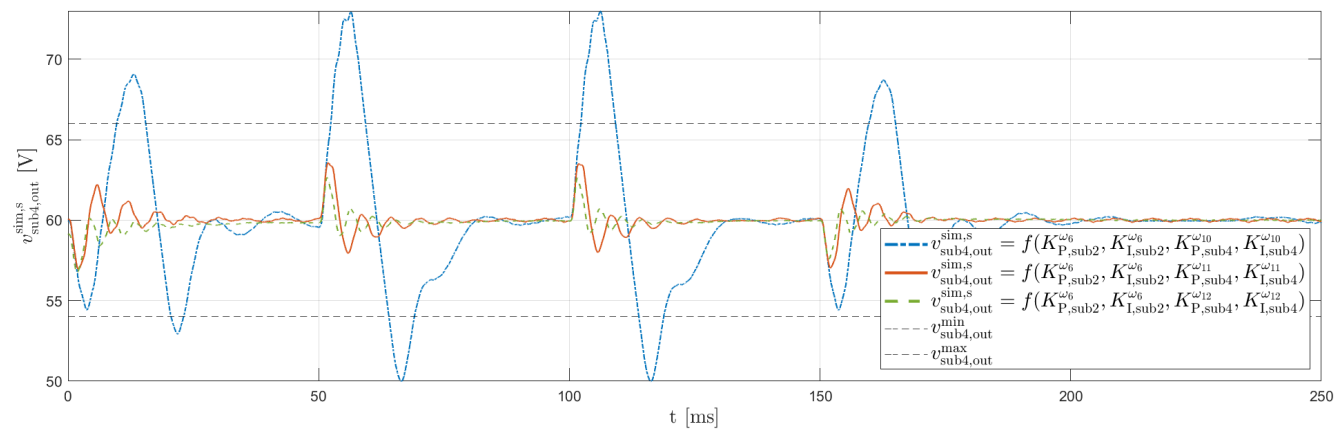

Figure 15. Sampled voltages in simulation model of sys 3 shown in Figure 1 with sub2 regulator gains at the $\omega_{6}$ from Table 3 and sub4 gains: $(\mathbf{a}, \mathbf{b})$ not optimised - the same as in the case of independently operating sys 2 at $\omega_{7} . . \omega_{9} ;(\mathbf{c}, \mathbf{d})$ optimised-according to the $K_{\mathrm{P}}$ and $K_{\mathrm{I}}$ located at $\omega_{10} . . \omega_{12}$ to satisfy the tuning criterions shown in Figure 12e. The results are for sys 3 constant resistive load, $R_{\text {out }}=33.3 \Omega$, corresponding to the output power of $108 \mathrm{~W}$. The input voltage profile, $v_{\mathrm{sys} 3, \mathrm{in}}^{\mathrm{sim}, \mathrm{s}}=v_{\mathrm{sub} 1, \mathrm{in}}^{\mathrm{sim}, \mathrm{s}}$, was the same as shown in Figure 13a. They are to be compared with mathematically calculated equivalents shown in Figure 14. Corresponding currents can be seen in Figure 16.

(a)

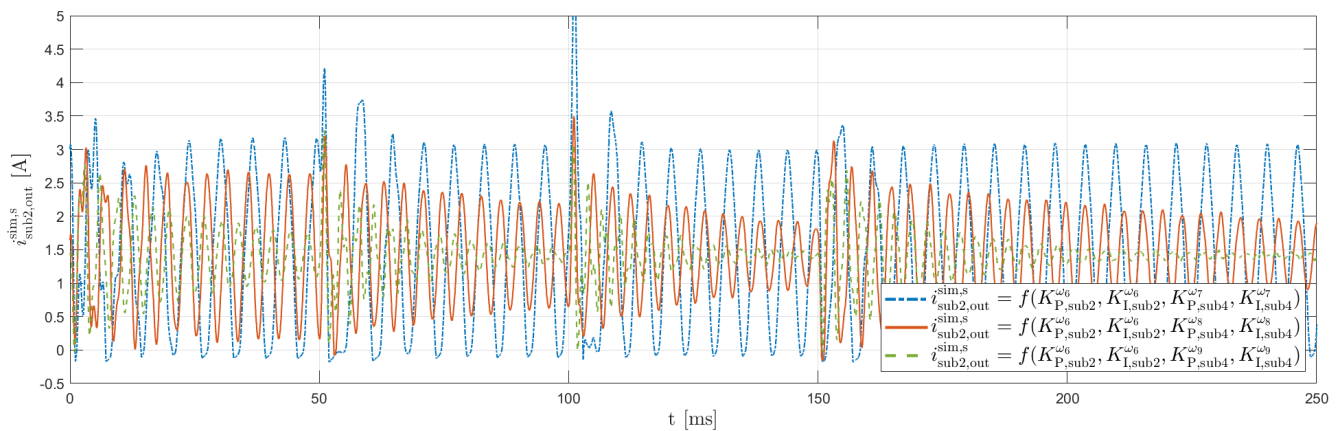

(b)

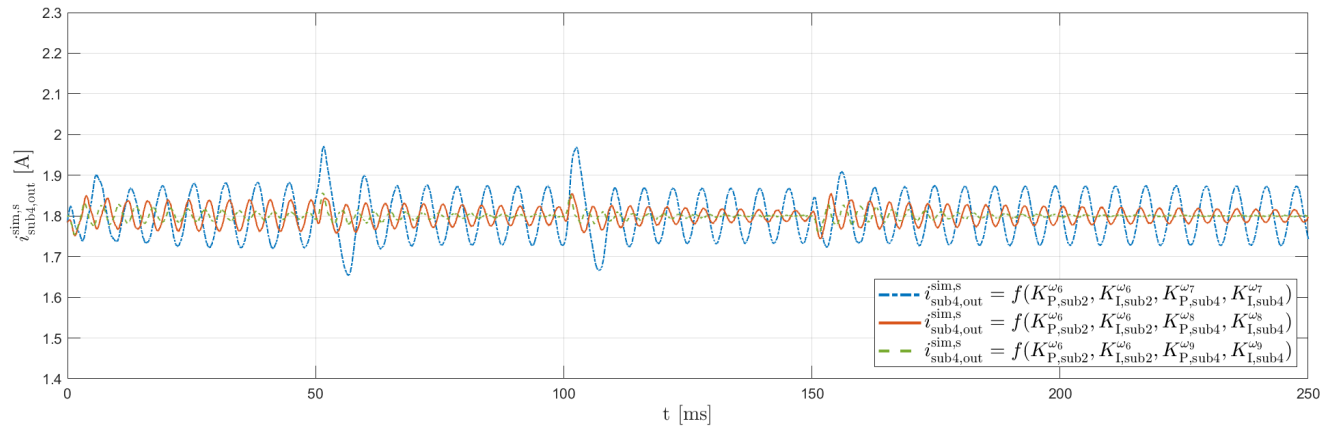

(c)

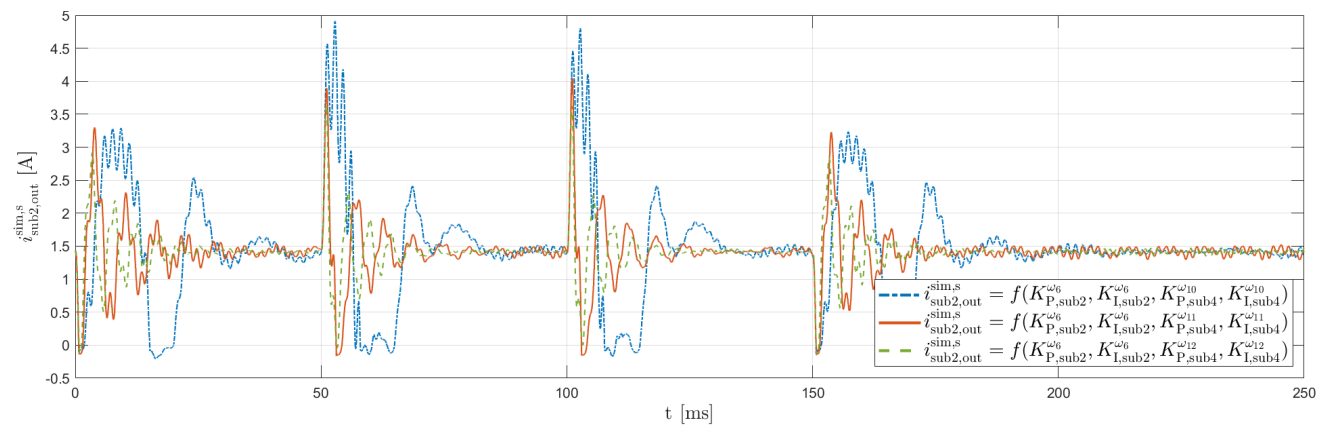

Figure 16. Cont. 
(d)

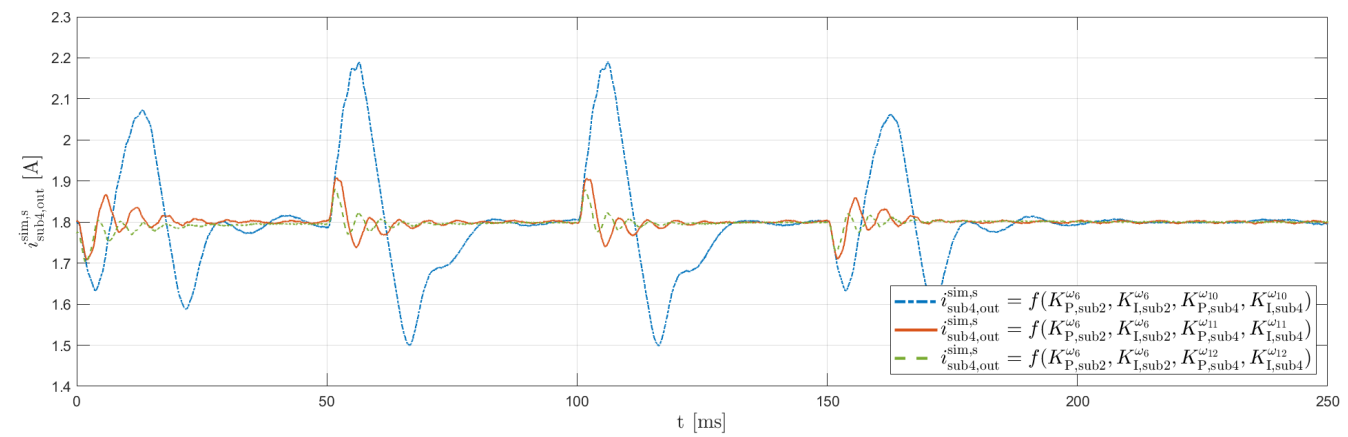

Figure 16. Sampled currents in simulation model of sys 3 shown in Figure 1. The plots correspond directly to the voltages shown in Figure 15.

\section{Conclusions}

The effectiveness of the $\mathfrak{D}$-decomposition technique merged with the selected control margins for the PI compensators gains selection has been shown in this article. It has been performed at a power electronics system level with the controlled circuits' passive filters included. The gains are calculated based on the GMPM criterion. Solutions are shown in an easy-to-interpret format as $K_{\mathrm{I}}=f\left(K_{\mathrm{P}}\right)$ functions. They are inscribed into the global stability boundaries for better visualization.

The effectiveness is concerned with the selection of single circuit gains and optimization of gains in an additional circuit connected to the present circuit. The gains have been derived step-by-step to explicitly show dependencies between two cascaded systems. First, the two systems were tuned independently. After, optimisation of the gains of the output system was applied to the frozen gains of the input system. The obtained results clearly show the necessity for an overall system-level gains optimisation.

All work is based on mathematical and simulation modelling. Major parts of the simulation model have been verified experimentally. All necessary transfer functions are included and explained in the article.

The PES control design has been considered on the basis of two boost-converter PI output voltage regulators. At this stage of research, based on the obtained results, one initial recommendation for the output system gains selection could be formulated as follows: use gains located in the higher frequency range of the calculated region. Nevertheless, further tests and investigations are required before this general recommendation can be verified.

Author Contributions: Conceptualization, methodology, validation, formal analysis, resources, funding acquisition, K.N. and R.N.; software, investigation, data curation, K.N.; writing-original draft preparation, R.N.; writing-review and editing, R.N., K.N. and R.L.; visualization, K.N.; supervision, R.N. and R.L.; project administration, R.L. All authors have read and agreed to the published version of the manuscript.

Funding: This research received no external funding.

Institutional Review Board Statement: Not applicable.

Informed Consent Statement: Not applicable.

Data Availability Statement: Not applicable.

Conflicts of Interest: The authors declare no conflict of interest.

\section{References}

1. Bose, B.K. Power Electronics in Smart Grid and Renewable Energy Systems. Proc. IEEE 2017, 105, 2007-2010. [CrossRef]

2. Huber, J.E.; Kolar, J.W. Applicability of Solid-State Transformers in Today's and Future Distribution Grids. IEEE Trans. Smart Grid 2019, 10, 317-326. [CrossRef] 
3. Huber, J.E.; Rothmund, D.; Wang, L.; Kolar, J.W. Full-ZVS modulation for all-SiC ISOP-type isolated front end (IFE) solid-state transformer. In Proceedings of the 2016 IEEE Energy Conversion Congress and Exposition (ECCE), Milwaukee, WI, USA, 18-22 September 2016; pp. 1-8.

4. Kumar, V.I.; Kapat, S. A Digitally Current Mode Controlled Non-Inverting Buck-Boost Converter for Fast Voltage Transitions. In Proceedings of the 2021 IEEE Applied Power Electronics Conference and Exposition (APEC), Phoenix, AZ, USA, 14-17 June 2021; pp. 2520-2525.

5. Allani, M.Y.; Riahi, J.; Vergura, S.; Mami, A. FPGA-Based Controller for a Hybrid Grid-Connected PV/Wind/Battery Power System with AC Load. Energies 2021, 14, 2108. [CrossRef]

6. Ogata, K. Modern Control Engineering, 5th ed.; Prentice Hall: Upper Saddle River, NJ, USA, 2010.

7. Yfoulis, C. An MPC Reference Governor Approach for Enhancing the Performance of Precompensated Boost DC-DC Converters. Energies 2019, 12, 563. [CrossRef]

8. Ray, P.K.; Das, S.R.; Mohanty, A. Fuzzy-Controller-Designed-PV-Based Custom Power Device for Power Quality Enhancement. IEEE Trans. Energy Convers. 2019, 34, 405-414. [CrossRef]

9. Orlowska-Kowalska, T.; Szabat, K. Optimization of fuzzy-logic speed controller for DC drive system with elastic joints. IEEE Trans. Ind. Appl. 2004, 40, 1138-1144. [CrossRef]

10. Vasantharaj, S.; Indragandhi, V.; Subramaniyaswamy, V.; Teekaraman, Y.; Kuppusamy, R.; Nikolovski, S. Efficient Control of DC Microgrid with Hybrid PV-Fuel Cell and Energy Storage Systems. Energies 2021, 14, 3234. [CrossRef]

11. Åström, K.J.; Wittenmark, B. Adaptive Control, 2nd ed.; Addison-Wesley: Boston, MA, USA, 1995.

12. Pratama, S.C.; Susanto, E.; Wibowo, A.S. Design and implementation of water level control using gain scheduling PID back calculation integrator Anti Windup. In Proceedings of the 2016 International Conference on Control, Electronics, Renewable Energy and Communications (ICCEREC), Bandung, Indonesia, 13-15 September 2016; pp. 101-104.

13. Niejmark, J.I. Ob opriedielenji znaczenij paramietrow, pri kotorych sistiema awtomaticzeskogo riegulirowanja ustojcziwa. Awtomatika Telemiechanika 1948, 9, 190-203.

14. Shafiei, Z.; Shenton, A.T. Relative stability for open-loop stable and unstable discrete control systems with perturbed or adjustable parameters. In Proceedings of the 1999 European Control Conference (ECC), Karlsruhe, Germany, 31 August-3 September 1999; pp. 2180-2185.

15. Onat, C.; Hamamci, S.E.; Obuz, S. A Practical PI Tuning Approach For Time Delay Systems. IFAC Proc. Vol. 2012, 45, 102-107. [CrossRef]

16. Wróbel, K.; Nalepa, R.; Najdek, K.; Szabat, K. Design of the control structure for two-mass system with help of the Ddecomposition technique. In Proceedings of the 2021 IEEE 19th International Power Electronics and Motion Control Conference (PEMC), Gliwice, Poland, 25-29 April 2021; pp. 711-716.

17. Najdek, K.; Nalepa, R. Use of the D-decomposition technique for gains selection of the Dual Active Bridge converter output voltage regulator. Przeglad Elektrotechniczny 2019, 95, 268-273. [CrossRef]

18. Najdek, K.; Nalepa, R. The Frequency- and the Time-Domain Design of a Dual Active Bridge Converter Output Voltage Regulator Based on the D-Decomposition Technique. IEEE Access 2021, 9, 71388-71405. [CrossRef]

19. Li, H.; Zhang, X.; Yang, S.; Li, E.; Hong, J. Multi-Objective Controller Design of IPMSM Drives Based on DTD D-Partition Method Considering Parameters Uncertainties. IEEE Trans. Energy Convers. 2019, 34, 1052-1062. [CrossRef]

20. Schrödel, F.; Liu, H.; Elghandour, R.; Abel, D. Lyapunov-based stability region computation approach. In Proceedings of the 2015 European Control Conference (ECC), Linz, Austria, 15-17 July 2015; pp. 3009-3014.

21. Najdek, K.; Nalepa, R.; Szabat, K. Selection of Controller Parameters of a Two-Mass Drive System Using the D-Decomposition Technique. In Proceedings of the IECON 2019-45th Annual Conference of the IEEE Industrial Electronics Society, Lisbon, Portugal, 14-17 October 2019; pp. 1308-1313.

22. Phattanasak, M.; Gavagsaz-Ghoachani, R.; Martin, J.; Pierfederici, S.; Nahid-Mobarakeh, B.; Riedinger, P. Lyapunov-based control and observer of a boost converter with LC input filter and stability analysis. In Proceedings of the International Conference on Electrical Systems for Aircraft, Railway, Ship Propulsion and Road Vehicles \& International Transportation Electrification Conference (ESARS-ITEC), Toulouse, France, 2-4 November 2016; pp. 1-6.

23. Tian, Y.; Loh, P.C.; Chen, Z.; Deng, F.; Hu, Y. Impedance interactions in bidirectional cascaded converter. IET Power Electron. 2016, 9, 2482-2491. [CrossRef]

24. Méndez-Elizondo, S.; Morales-Saldaña, J.; Reyes-Portillo, I.; Peña-Gallardo, R.; Netzahuatl-Huerta, E. Robustness Study of a DC Nanogrid Based on a Distributed Generation System. In Proceedings of the 2020 IEEE International Autumn Meeting on Power, Electronics and Computing (ROPEC), Ixtapa, Mexico, 4-6 November 2020; pp. 1-6.

25. Vesti, S.; Oliver, J.A.; Prieto, R.; Cobos, J.A.; Suntio, T. Simplified small-signal stability analysis for optimized power system architecture. In Proceedings of the IEEE Applied Power Electronics Conference and Exposition (APEC), Long Beach, CA, USA, 17-21 March 2013; pp. 1702-1708.

26. Garcia-Rodriguez, V.H.; Silva-Ortigoza, R.; Hernández-Márquez, E.; Garcia-Sánchez, J.R.; Taud, H. DC/DC Boost Converter-Inverter as Driver for a DC Motor: Modeling and Experimental Verification. Energies 2018, 11, 2044. [CrossRef]

27. Son, Y.I.; Kim, I.H. Complementary PID Controller to Passivity-Based Nonlinear Control of Boost Converters With Inductor Resistance. IEEE Trans. Control Syst. Technol. 2012, 20, 826-834. [CrossRef] 
28. Licea, M.A.R.; Pinal, F.J.P.; Gutiérrez, A.I.B.; Ramírez, C.A.H.; Perez, J.C.N. A Reconfigurable Buck, Boost, and Buck-Boost Converter: Unified Model and Robust Controller. Math. Probl. Eng. 2018, 2018. [CrossRef]

29. Ioinovici, A. Modeling DC-DC Converters. In Power Electronics and Energy Conversion Systems; Wiley: Chichester, UK; Hoboken, NJ, USA, 2013; pp. 161-368.

30. Cheng, Z.; Li, Z.; Li, S.; Gao, J.; Si, J.; Das, H.S.; Dong, W. A novel cascaded control to improve stability and inertia of parallel buck-boost converters in DC microgrid. Electr. Power Energy Syst. 2020, 119, 105950. [CrossRef]

31. Baghaee, H.R.; Mlakić, D.; Nikolovski, S.; Dragicčvić, T. Anti-Islanding Protection of PV-Based Microgrids Consisting of PHEVs Using SVMs. IEEE Trans. Smart Grid 2020, 11, 483-500. [CrossRef]

32. Nalepa, R.; Najdek, K.; Strong, B. Hybrid Tuning of a Boost Converter PI Voltage Compensator by Means of the Genetic Algorithm and the D-Decomposition. Energies 2021, 14, 173. [CrossRef]

33. Riccobono, A.; Mirz, M.; Ferdowsi, M.; Monti, A. Modern Control of DC-Based Power Systems: A Problem-Based Approach; Academic Press: Cambridge, MA, USA, 2018.

34. Vesti, S.; Suntio, T.; Oliver, J.A.; Prieto, R.; Cobos, J.A. Impedance-Based Stability and Transient-Performance Assessment Applying Maximum Peak Criteria. IEEE Trans. Power Electron. 2013, 28, 2099-2104. [CrossRef] 